\title{
FAST DETERMINISTIC PRICING OF OPTIONS ON LÉVY DRIVEN ASSETS*
}

\author{
Ana-Maria Matache ${ }^{1}$, Tobias von PetersdorfF ${ }^{2}$ and Christoph Schwab $^{3}$
}

\begin{abstract}
Arbitrage-free prices $u$ of European contracts on risky assets whose log-returns are modelled by Lévy processes satisfy a parabolic partial integro-differential equation (PIDE) $\partial_{t} u+\mathcal{A}[u]=0$. This PIDE is localized to bounded domains and the error due to this localization is estimated. The localized PIDE is discretized by the $\theta$-scheme in time and a wavelet Galerkin method with $N$ degrees of freedom in log-price space. The dense matrix for $\mathcal{A}$ can be replaced by a sparse matrix in the wavelet basis, and the linear systems in each implicit time step are solved approximatively with GMRES in linear complexity. The total work of the algorithm for $M$ time steps is bounded by $O\left(M N(\log (N))^{2}\right)$ operations and $O(N \log (N))$ memory. The deterministic algorithm gives optimal convergence rates (up to logarithmic terms) for the computed solution in the same complexity as finite difference approximations of the standard Black-Scholes equation. Computational examples for various Lévy price processes are presented.
\end{abstract}

Mathematics Subject Classification. 65N30, 60J75.

Received: February 27, 2003.

\section{INTRODUCTION}

Since the seminal paper [8], the pricing of options by means of partial differential equations has become standard practice in quantitative finance, either by means of explicit solution formulas for the heat equation (e.g. $[21,23,25])$ in the case of European vanillas or by numerical methods in the case of American or Barrier options.

In recent years, awareness of the shortcomings of the Black-Scholes model has increased and more general models for the stochastic dynamics of the risky asset have been proposed: we mention only stochastic volatility models and "stochastic clocks". The latter lead to price processes with a jump component: the Wiener process

Keywords and phrases. Parabolic partial integro-differential equations, Lévy processes, Markov processes, Galerkin finite element method, wavelet, matrix compression, GMRES.

* Research was supported by Credit Suisse Group, Swiss Re and UBS AG through RiskLab, Switzerland under the RiskLab project Fast Deterministic Valuations for Assets Driven by Lévy Processes and performed while the 2nd author visited the Seminar for Applied Mathematics of ETH Zürich in 2001. C. Schwab was supported in part under the IHP network Breaking Complexity of the EC (contract number HPRN-CT-2002-00286) with support by the Swiss Federal Office for Science and Education under grant No. BBW 02.0418.

1 RiskLab and Seminar for Applied Mathematics, ETH-Zentrum, 8092 Zürich, Switzerland.

2 Department of Mathematics, University of Maryland, College Park, MD 20742, USA.

3 Seminar for Applied Mathematics, ETH-Zentrum, 8092 Zürich, Switzerland. e-mail: schwab@sam.math.ethz.ch 
from the Black-Scholes model is replaced by a Lévy process (see e.g. $[3,9,10,12,15,18,27,28,31,35-37]$ and the references therein and $[7,38]$ for fundamentals on Lévy processes).

Abandoning the Wiener process as price process renders the market in the model incomplete and the martingale measure in the pricing problem non-unique. After selection of an equivalent martingale measure $\mathbb{Q}$ the asset pricing problem becomes once again the problem of solving a deterministic equation. This equation is a parabolic integro-differential equation (PIDE) with non-integrable kernel if the jump activity of the Lévy process is infinite.

In case of European vanillas and in logarithmic price, this equation is posed on the whole real line. The justification, numerical analysis and rigorous derivation of efficient solution algorithms for this PIDE is the purpose of the present paper. Its outline is as follows: after brief recapitulation of the Black-Scholes model of asset pricing, and in particular of the functional setting which accommodates exponentially growing payoff functions we turn in Section 3 to the PIDE for pricing options on Lévy driven assets. We establish its well-posedness in spaces of possibly exponentially growing solutions and give a suitable variational formulation.

Section 4 is devoted to the truncation of the PIDE to a bounded domain - an essential step for numerical simulation as well as for modeling certain types of contracts. Due to the jump part of the Lévy process, this localization cannot be effected by simple restriction to the bounded domain plus suitable local boundary conditions, but must take into account the pay-off beyond the computational domain. We show how to do this so that the localization error decays exponentially with the size of the truncation domain; contrary to earlier work in the Black-Scholes case [22] the proof does not use the maximum principle, but rather a priori estimates in exponentially weighted spaces.

Section 5 is devoted to our solution algorithm - the $\theta$-scheme for time-stepping and a wavelet-Galerkin discretization of the integro-differential operator. We show that the solution algorithm has the same asymptotic complexity as the Finite Element Method (FEM) for the Black-Scholes equation. Finally, we present numerical examples of Lévy pricing - European vanillas under Variance Gamma and CGMY-processes with finite and infinite activity can be handled by our approach in a unified fashion.

Let us briefly comment on how our approach compares with Fourier techniques [11,12]. These methods require the characteristic function of the process and allow, via Fast Fourier Transform (FFT), the efficient evaluation of the jump operator. Unlike the Fourier Transform, wavelets are well localized also in price space which allows to treat barrier and American contracts; moreover, wavelets allow to compress finite intensity jump operators to sublinear complexity.

The wavelet approach requires the distributional kernel of the infinitesimal generator of the Lévy process, i.e., the inverse Fourier transform of the characteristic function. It allows to handle barrier, touch-and-out or no-touch type contracts deterministically, i.e. without Monte-Carlo techniques. It also allows to price American puts on Lévy driven underlying [30] and, more importantly, also accommodates more general infinite activity Markovian processes for the log-returns of the risky assets where the jump-measures are not translation invariant. The present results have been announced in [29].

\section{Pricing European vanillas in the Black-Scholes Setting}

Our option pricing algorithm will be developed in a variational framework. We present the necessary function spaces first for a Black-Scholes market [8], following [25].

Classical option pricing theory of Black and Scholes relies on the fact that the pay-off of every contingent claim can be duplicated by a portfolio consisting of investments in the underlying stock and in a bond paying a riskless rate of interest. The model of Black and Scholes consists of one risky asset, a share with spot price $S_{t}$ at time $t$ and a riskless asset with spot $S_{t}^{0}$ at time $t$ satisfying the following ordinary differential equation

$$
\mathrm{d} S_{t}^{0}=r S_{t}^{0} \mathrm{~d} t
$$


with $r>0$ being the riskless interest rate. The price of the risky asset is modelled by the following stochastic differential equation

$$
\mathrm{d} S_{t}=S_{t}\left(\mu \mathrm{d} t+\sigma \mathrm{d} B_{t}\right)
$$

with $\mu, \sigma$ being constants and $B_{t}$ the standard brownian motion built on a probability space $(\Omega, \mathcal{F}, P)$. We denote by $\left(\mathcal{F}_{t}\right)_{t}$ its natural filtration. It is well-known, see e.g. [25], that there exists a unique probability measure $\mathbb{Q}$ under which the discounted stock price $\tilde{S}_{t}:=\mathrm{e}^{-r t} S_{t}$ is a martingale and any option defined by a non-negative, $\mathcal{F}_{T}$-measurable random variable $g$ is replicable and the value at time $t<T$ of any replicating portfolio is given by

$$
f\left(t, S_{t}\right)=E_{\mathbb{Q}}\left[\mathrm{e}^{-r(T-t)} g\left(S_{T}\right) \mid \mathcal{F}_{t}\right]
$$

\subsection{Black-Scholes equation}

To present the Black-Scholes (BS) equation and its variational formulation, we focus exemplarily on European call options with pay-off $(S-K)_{+}:=\max \{S-K, 0\}$ but emphasize that our framework accommodates pay-off functions in $L_{l o c}^{2}\left(\mathbb{R}_{+}\right)$with polynomial growth as $|S| \rightarrow \infty$.

The price $f\left(t, S_{t}\right)$ has to satisfy the BS equation

$$
\frac{\partial f}{\partial t}+\frac{\sigma^{2}}{2} S^{2} \frac{\partial^{2} f}{\partial S^{2}}+r S \frac{\partial f}{\partial S}-r f=0 \quad \text { in } \quad(0, T) \times(0, \infty)
$$

together with the terminal condition at maturity

$$
f(T, S)=(S-K)_{+}
$$

The BS equation (2.1) degenerates at $S=0$. To remove the degeneracy, we change to logarithmic return price $x=\log (S)$ and write the BS equation (2.1)-(2.2) for $u(t, x):=f\left(t, \mathrm{e}^{x}\right)$

$$
\begin{aligned}
\frac{\partial u}{\partial t}+\frac{\sigma^{2}}{2} \frac{\partial^{2} u}{\partial x^{2}}+\left(r-\frac{\sigma^{2}}{2}\right) \frac{\partial u}{\partial x}-r u & =0 \quad \text { in } \quad(0, T) \times \mathbb{R} \\
u(T, x) & =h(x):=\left(\mathrm{e}^{x}-K\right)_{+} .
\end{aligned}
$$

In the time to maturity $\tau=T-t,(2.3)$ for $w(\tau, x)=u(T-\tau, x)$ reads:

$$
\begin{aligned}
\frac{\partial w}{\partial \tau}-\frac{\sigma^{2}}{2} \frac{\partial^{2} w}{\partial x^{2}}-\left(r-\frac{\sigma^{2}}{2}\right) \frac{\partial w}{\partial x}+r w & =0 \quad \text { in } \quad(0, T) \times \mathbb{R} \\
w(0, x) & =h(x) .
\end{aligned}
$$

\subsection{Variational formulation}

We derive the variational formulation to (2.4). We observe that the pay-off function $h$ in $(2.3)$, (2.4) does not belong to $L^{2}(\mathbb{R})$. Moreover, since we switched to logarithmic price, the payoff grows exponentially at infinity, therefore we cannot use standard Sobolev spaces as function spaces for this problem. We introduce weighted Sobolev spaces to account for the exponential growth of solutions at infinity, following [21].

For $\nu \in \mathbb{R}$ we define the weighted Sobolev space $H_{\nu}^{1}(\mathbb{R})$ by

$$
H_{\nu}^{1}(\mathbb{R}):=\left\{v \in L_{\text {loc }}^{1}(\mathbb{R}) \mid v \mathrm{e}^{\nu|x|}, v^{\prime} \mathrm{e}^{\nu|x|} \in L^{2}(\mathbb{R})\right\}
$$

Similarly, $L_{\nu}^{2}(\mathbb{R}):=\left\{v \in L_{\text {loc }}^{1}(\mathbb{R}) \mid v \mathrm{e}^{\nu|x|} \in L^{2}(\mathbb{R})\right\}$. With this notation, the pay-off function $h$ in $(2.4)$ belongs to $H_{-\mu}^{1}(\mathbb{R})$ for any $\mu>1$. 
In order to cast (2.4) in a variational form we consider a test function $v \in C_{0}^{\infty}(\mathbb{R})$ and we multiply (2.4) by $v \mathrm{e}^{-2 \nu|x|}$, with $\nu \in \mathbb{R}$ arbitrary, fixed. By integration by parts over $\mathbb{R}$ we obtain

$$
\begin{aligned}
\frac{\mathrm{d}}{\mathrm{d} \tau} \int_{\mathbb{R}} w(\tau, & x) v(x) \mathrm{e}^{-2 \nu|x|} \mathrm{d} x-\left(r-\frac{\sigma^{2}}{2}\right) \int_{\mathbb{R}} \frac{\partial w}{\partial x}(\tau, x) v(x) \mathrm{e}^{-2 \nu|x|} \mathrm{d} x \\
& +\frac{\sigma^{2}}{2} \int_{\mathbb{R}}\left\{\frac{\partial w}{\partial x}(\tau, x) \frac{\partial v}{\partial x}(x) \mathrm{e}^{-2 \nu|x|}-2 \nu \operatorname{sign}(x) \frac{\partial w}{\partial x}(\tau, x) v(x) \mathrm{e}^{-2 \nu|x|}\right\} \mathrm{d} x+\int_{\mathbb{R}} r w(\tau, x) v(x) \mathrm{e}^{-2 \nu|x|} \mathrm{d} x=0 .
\end{aligned}
$$

We define the bilinear form $a^{-\nu}(\cdot, \cdot): H_{-\nu}^{1}(\mathbb{R}) \times H_{-\nu}^{1}(\mathbb{R}) \rightarrow \mathbb{R}$ by

$$
\begin{aligned}
a^{-\nu}\left(v_{1}, v_{2}\right):=\frac{\sigma^{2}}{2} \int_{\mathbb{R}} v_{1}^{\prime}(x) v_{2}^{\prime}(x) \mathrm{e}^{-2 \nu|x|} \mathrm{d} x+\int_{\mathbb{R}} r v_{1}(x) v_{2}(x) \mathrm{e}^{-2 \nu|x|} \mathrm{d} x & -\int_{\mathbb{R}}\left(\nu \sigma^{2} \operatorname{sign}(x)+\left(r-\frac{\sigma^{2}}{2}\right)\right) v_{1}^{\prime}(x) v_{2}(x) \mathrm{e}^{-2 \nu|x|} \mathrm{d} x .
\end{aligned}
$$

With $\mu>1$ the variational formulation to $(2.4)$ reads:

Given $h \in H_{-\mu}^{1}(\mathbb{R})$, find $w \in L^{2}\left(0, T ; H_{-\mu}^{1}(\mathbb{R})\right) \cap H^{1}\left(0, T ;\left(H_{-\mu}^{1}(\mathbb{R})\right)^{*}\right)$ such that

$$
\begin{aligned}
\frac{\mathrm{d}}{\mathrm{d} \tau}(w(\tau, \cdot), v)_{L_{-\mu}^{2}(\mathbb{R})}+a^{-\mu}(w(\tau, \cdot), v(\cdot)) & =0 \quad \forall v \in H_{-\mu}^{1}(\mathbb{R}) \\
w(0, x) & =h(x) .
\end{aligned}
$$

To prove existence and uniqueness for the solution of (2.6), we analyze the properties of the bilinear form $a^{-\nu}(\cdot, \cdot)$ with respect to the weighted Sobolev spaces $H_{-\nu}^{1}(\mathbb{R})$ for arbitrary $\nu \in \mathbb{R}$.

Proposition 2.1. Let $\nu \in \mathbb{R}$ be arbitrary, fixed.

1. The bilinear form $a^{-\nu}(\cdot, \cdot): H_{-\nu}^{1}(\mathbb{R}) \times H_{-\nu}^{1}(\mathbb{R}) \rightarrow \mathbb{R}$ is continuous, i.e., there exists a constant $M>0$ such that

$$
\left|a^{-\nu}(u, v)\right| \leq M\|u\|_{H_{-\nu}^{1}(\mathbb{R})}\|v\|_{H_{-\nu}^{1}(\mathbb{R})} \quad \forall u, v \in H_{-\nu}^{1}(\mathbb{R}) .
$$

2. There exists $\lambda_{0}>0$ depending on $\nu$ such that for all $\lambda>\lambda_{0}$ the new bilinear form $a^{-\nu}(\cdot, \cdot)+\lambda(\cdot, \cdot)_{L_{-\nu}^{2}}(\mathbb{R})$ is coercive, i.e., there exists $\alpha>0$ such that for all $\lambda>\lambda_{0}$ it holds: $a^{-\nu}(u, u)+\lambda\|u\|_{L_{-\nu}^{2}(\mathbb{R})}^{2} \geq$ $\alpha\|u\|_{H_{-\nu}^{1}(\mathbb{R})}^{2} \forall u \in H_{-\nu}^{1}(\mathbb{R})$.

Proof. Take $v_{1}=v_{2}=u$ in the definition $(2.5)$ of the bilinear form $a^{-\nu}(\cdot, \cdot)$. Then, there exist some constants $\gamma>0, \beta \geq 0$ such that for all $u \in H_{-\nu}^{1}(\mathbb{R})$ it holds

$$
\begin{aligned}
a^{-\nu}(u, u) & =\frac{\sigma^{2}}{2}\left\|u^{\prime} \mathrm{e}^{-\nu|x|}\right\|_{L^{2}(\mathbb{R})}^{2}-\int_{\mathbb{R}}\left(\nu \operatorname{sign}(x) \sigma^{2}+r-\frac{\sigma^{2}}{2}\right) u^{\prime}(x) u(x) \mathrm{e}^{-2 \nu|x|} \mathrm{d} x+r\left\|u \mathrm{e}^{-\nu|x|}\right\|_{L^{2}(\mathbb{R})}^{2} \\
& \geq \gamma\left\|u^{\prime} \mathrm{e}^{-\nu|x|}\right\|_{L^{2}(\mathbb{R})}^{2}-\beta\left\|u \mathrm{e}^{-\nu|x|}\right\|_{L^{2}(\mathbb{R})}^{2}
\end{aligned}
$$

Choosing now $\lambda_{0}>\beta$ we obtain 2. The assertion 1. follows from the Cauchy-Schwarz inequality. 
Remark 2.2. Without loss of generality we assume from now on that $a^{-\nu}(\cdot, \cdot)$ is coercive with coercivity constant $\alpha>0$. Indeed, by the transformation $v(\tau, x)=\mathrm{e}^{-\lambda \tau} w(\tau, x)$ the problem for $v$ reads

$$
\begin{aligned}
\frac{\partial v}{\partial \tau}-\frac{\sigma^{2}}{2} \frac{\partial^{2} v}{\partial x^{2}}+\left(\frac{\sigma^{2}}{2}-r\right) \frac{\partial v}{\partial x}+(r+\lambda) v & =0 \quad \in(0, T) \times \mathbb{R} \\
v(0, x) & =h(x)
\end{aligned}
$$

and the corresponding bilinear form $a^{-\nu}(\cdot, \cdot)+\lambda(\cdot, \cdot)_{L_{-\nu}^{2}(\mathbb{R})}$ is by Proposition 2.12 . for all $\lambda>\lambda_{0}$ coercive.

\subsection{Functional setting}

\subsubsection{Abstract parabolic problems}

We give an abstract functional setting for the existence and continuous dependence of weak solutions of parabolic problems which will be used for (2.3) but also later for Lévy processes. It is based on the following Gelfand triple:

$$
V \hookrightarrow H \cong H^{*} \hookrightarrow V^{*}
$$

where $V$ and $H$ are separable Hilbert spaces and $\hookrightarrow$ means dense, but possibly non-compact embedding. We assume $\mathcal{A} \in \mathcal{L}\left(V, V^{*}\right)$ to be an elliptic "spatial differential" operator given in the weak form

$$
\langle\mathcal{A} u, v\rangle_{V^{*} \times V}=a(u, v), \quad \forall u, v \in V
$$

where the bilinear form $a(\cdot, \cdot): V \times V \rightarrow \mathbb{R}$ is continuous and satisfies a Gårding inequality: there are constants $C_{i} \geq 0$ such that

$$
\begin{aligned}
& \forall u \in V, v \in V:|a(u, v)| \leq C_{0}\|u\|_{V}\|v\|_{V} \\
& \forall u \in V: a(u, u) \geq C_{1}\|u\|_{V}^{2}-C_{2}\|u\|_{H}^{2} .
\end{aligned}
$$

In the triple (2.8) we consider the abstract parabolic problem

$$
u^{\prime}(t)+\mathcal{A} u(t)=f \quad \text { in } V^{*}, \quad t \in(0, T), \quad u(0)=u_{0} \in H
$$

Without loss of generality we may assume that $C_{2}=0$ in $(2.11)$, since by the substitution

$$
w=\mathrm{e}^{-C_{2} t} u
$$

we obtain that $w$ solves

$$
\begin{aligned}
w^{\prime}(t)+\mathcal{A} w(t)+C_{2} w(t) & =\mathrm{e}^{-C_{2} t} f \quad \text { in } V^{*}, \quad t \in(0, T) \\
w(0) & =u_{0} \quad \text { in } H
\end{aligned}
$$

and $\mathcal{A}+C_{2} I$ is by $(2.11)$ positive.

In our treatment of Lévy processes we need a general parabolic existence result in the triple (2.8).

Theorem 2.3. Assume $(2.8), \mathcal{A} \in \mathcal{L}\left(V, V^{*}\right)$ and that the bilinear form $a(\cdot, \cdot)$ in $(2.9)$ satisfies (2.11) with $C_{2}=0$. Then

1. $\mathcal{A} \in \mathcal{L}\left(V, V^{*}\right)$ is an isomorphism.

2. $-\mathcal{A}$ is the infinitesimal generator of a bounded analytic $C^{0}$-semi-group $E(t)$ in $V^{*}$. 
3. For $u_{0} \in H$ and $f \in L^{2}\left(0, T ; V^{*}\right)$, the evolution problem (2.14) has a unique solution $u \in L^{2}(0, T ; V) \cap$ $H^{1}\left(0, T ; V^{*}\right)$ which can be represented as

$$
u(t)=E(t) u_{0}+\int_{0}^{t} E(t-s) f(s) \mathrm{d} x .
$$

Moreover, the following a priori estimate (cf. e.g., [26]) holds

$$
\|u\|_{L^{2}(0, T ; V)}+\left\|u^{\prime}\right\|_{L^{2}\left(0, T ; V^{*}\right)}+\|u\|_{C([0, T] ; H)} \leq C\left(\left\|u_{0}\right\|_{H}+\|f\|_{L^{2}\left(0, T ; V^{*}\right)}\right) .
$$

Proof. We assume first that $f=0$ and proceed in several steps.

Step 1. $\mathcal{A}$ is a closed operator, since the graph norm $\|u\|_{\mathcal{A}}:=\|\mathcal{A} u\|_{V^{*}}+\|i u\|_{V^{*}}$, with $V \stackrel{i}{\hookrightarrow} V^{*}$, is an equivalent norm for $X$.

Step 2. For all $\lambda \in \mathbb{C}$, with $\operatorname{Re} \lambda>0$,

$$
(u, v) \mapsto((\mathcal{A}+\lambda I) u, v)_{V^{*} \times V}
$$

is also positive and there holds

$$
\left\|(\lambda I+\mathcal{A})^{-1} g\right\|_{V} \leq \frac{1}{\alpha}\|g\|_{V^{*}}, \quad\left\|(\lambda I+\mathcal{A})^{-1} g\right\|_{V^{*}} \leq \frac{1}{|\lambda|}\left(\frac{M}{\alpha}+1\right)\|g\|_{V^{*}} .
$$

Step 3. By Step 1. and Step 2. and since $0 \in \rho(-\mathcal{A})$ it follows that there exists $0<\delta<\pi / 2$ and there exists $C>0$ such that

and

$$
\rho(-\mathcal{A}) \supset \Sigma_{\delta}:=\{\lambda \in \mathbb{C}:|\arg \lambda|<\pi / 2+\delta\} \cup\{0\}
$$

$$
\left\|(\lambda I+\mathcal{A})^{-1}\right\|_{\mathcal{L}\left(V^{*}, V^{*}\right)} \leq \frac{C}{|\lambda|} \quad \forall \lambda \in \Sigma_{\delta}, \lambda \neq 0 .
$$

Indeed, by (2.16), $\left\|(\lambda I+\mathcal{A})^{-1}\right\|_{\mathcal{L}\left(V^{*}, V^{*}\right)} \leq C /|\operatorname{Im} \lambda|$ for all $\operatorname{Re} \lambda>0$. For $\bar{\lambda}=\xi+i \zeta$ with $\xi>0$, the Taylor expansion for $(\lambda I+\mathcal{A})^{-1}$ around $\bar{\lambda}$

$$
(\lambda I+\mathcal{A})^{-1}=\sum_{k=0}^{\infty}\left((\bar{\lambda} I+\mathcal{A})^{-1}\right)^{k+1}(\bar{\lambda}-\lambda)^{k}
$$

converges in $\mathcal{L}\left(V^{*}, V^{*}\right)$ for $\left\|(\bar{\lambda} I+\mathcal{A})^{-1}\right\|_{\mathcal{L}\left(V^{*}, V^{*}\right)}|\bar{\lambda}-\lambda| \leq q<1$. Choosing $\operatorname{Im} \lambda=\zeta$ we see that the series converges uniformly in $\mathcal{L}\left(V^{*}, V^{*}\right)$ for $|\xi-\operatorname{Re} \lambda| \leq q|\zeta| / C$. Since $\xi>0$ and $q \in(0,1)$ are arbitrary, $\rho(-\mathcal{A})$ contains all $\lambda \in \mathbb{C}$ with $\operatorname{Re} \lambda \leq 0$ and $|\operatorname{Re} \lambda| /|\operatorname{Im} \lambda|<1 / C$ and in particular $\rho(-\mathcal{A}) \supset\{\lambda \in \mathbb{C}:|\arg \lambda|<\pi / 2+\delta\}$ with $\delta=q \arctan (1 / C), 0<q<1$, and in this region we also have $\left\|(\lambda I+\mathcal{A})^{-1}\right\|_{\mathcal{L}\left(V^{*}, V^{*}\right)} \leq C /|\lambda|$.

By Theorems 1.7.7 and 2.5.2 in [33] it follows that $-\mathcal{A}$ is the infinitesimal generator of a uniformly bounded $C^{0}$-semigroup in $V^{*}$. Moreover, $E(t)$ can be extended to an analytic semigroup in the sector $\Delta_{\delta}=\{z \in \mathbb{C}$ : $|\arg z|<\delta\}$ and $\|E(t)\|_{\mathcal{L}\left(V^{*}, V^{*}\right)}$ is uniformly bounded in every closed subsector $\Delta_{\delta^{\prime}}, \delta^{\prime}<\delta$, of $\Delta_{\delta}$.

In the case $f \neq 0$, we use that the part of $u$ due to $f$ satisfies a Duhamel representation ([2], Prop. III.1.3.1) to conclude.

Remark 2.4. Elements of $\left(H_{-\nu}^{1}(\mathbb{R})\right)^{*}$ decay exponentially at infinity: consider $\phi \in H_{-\nu}^{1}(\mathbb{R})$ arbitrary, fixed and let $\phi_{n} \in H_{-\nu}^{1}(\mathbb{R}), n \in \mathbb{N}$ be given by $\phi_{n}(y):=\phi(y-n)$. Then for each $f \in\left(H_{-\nu}^{1}(\mathbb{R})\right)^{*}, \nu>0$, there is $C_{f}$ independent of $n$ with

$$
\forall n \in \mathbb{N} \quad\left|\left\langle f, \phi_{n}\right\rangle_{\left(H_{-\nu}^{1}(\mathbb{R})\right)^{*} \times H_{-\nu}^{1}(\mathbb{R})}\right| \leq C_{f} \mathrm{e}^{-\nu n}\|\phi\|_{H_{-\nu}^{1}(\mathbb{R})} .
$$




\subsubsection{Application to the Black-Scholes equation}

We apply Theorem 2.3 to the BS equation $(2.4)$ with $V=H_{-\nu}^{1}(\mathbb{R}), H=L_{-\nu}^{2}(\mathbb{R})$ and with

$$
\mathcal{A} u:=-\frac{\sigma^{2}}{2} \frac{\partial^{2} u}{\partial x^{2}}-\left(r-\frac{\sigma^{2}}{2}\right) \frac{\partial u}{\partial x}+r u
$$

Then the solution $w$ of (2.4) can be represented as

$$
w(\tau, \cdot)=E^{-\nu}(\tau) h,
$$

where $E^{-\nu}$ is the $C^{0}$ semigroup in $\left(H_{-\nu}^{1}(\mathbb{R})\right)^{*}$ with infinitesimal generator $\mathcal{A}$.

The case $r=0$. When $r=0$, i.e., $w$ solves

$$
\begin{aligned}
\frac{\partial w}{\partial \tau}-\frac{\sigma^{2}}{2} \frac{\partial^{2} w}{\partial x^{2}}+\frac{\sigma^{2}}{2} \frac{\partial w}{\partial x} & =0, \quad(\tau, x) \in(0, T) \times \mathbb{R} \\
w(0, x) & =h(x):=\left(\mathrm{e}^{x}-K\right)_{+} .
\end{aligned}
$$

By Proposition 2.1 and Theorem 2.3, given $h \in H_{-\zeta}^{1}(\mathbb{R}), \zeta>0,(2.4)$ admits a unique solution $w \in L^{2}\left(0, T ; H_{-\zeta}^{1}(\mathbb{R})\right) \cap H^{1}\left(0, T ;\left(H_{-\zeta}^{1}(\mathbb{R})\right)^{*}\right)$ and $(2.15)$ holds.

As second application of Theorem 2.3, we show now that $w(\tau, x)$ approaches the payoff $h(x)$ exponentially fast as $|x| \rightarrow \infty$ for $0<\tau<T$. To this end, we note that $\bar{w}:=w-h$ solves

$$
\frac{\partial \bar{w}}{\partial \tau}-\frac{\sigma^{2}}{2} \frac{\partial^{2} \bar{w}}{\partial x^{2}}+\frac{\sigma^{2}}{2} \frac{\partial \bar{w}}{\partial x}=f \quad \bar{w}(0, x)=0
$$

with $f:=\frac{\sigma^{2}}{2} K \delta_{\log (K)}$. Indeed, for $\mu>1, \mathcal{A} h \in\left(H_{-\mu}^{1}(\mathbb{R})\right)^{*}$ is given by

$$
\langle\mathcal{A} h, \varphi\rangle_{\left(H_{-\mu}^{1}(\mathbb{R})\right)^{*} \times H_{-\mu}^{1}(\mathbb{R})}=a^{-\mu}(h, \varphi) \quad \forall \varphi \in H_{-\mu}^{1}(\mathbb{R})
$$

and there holds

$$
\frac{\mathrm{d}}{\mathrm{d} \tau}(\bar{w}(\tau, \cdot), \varphi)_{L_{-\mu}^{2}(\mathbb{R})}+a^{-\mu}(\bar{w}(\tau, \cdot), \varphi)=-a^{-\mu}(h, \varphi) \quad \forall \varphi \in H_{-\mu}^{1}(\mathbb{R}) .
$$

By the definition of $a^{-\mu}(\cdot, \cdot)$ we obtain that the right hand side in (2.19) is given by

$$
-\frac{\sigma^{2}}{2} \int_{\log (K)}^{\infty} \mathrm{e}^{x} \varphi^{\prime}(x) \mathrm{e}^{-2 \mu|x|} \mathrm{d} x+\int_{\log (K)}^{\infty}\left(\mu \sigma^{2} \operatorname{sign}(x)-\frac{\sigma^{2}}{2}\right) \mathrm{e}^{x} \varphi(x) \mathrm{e}^{-2 \mu|x|} \mathrm{d} x=\frac{\sigma^{2}}{2} K \mathrm{e}^{-2 \mu|\log (K)|} \varphi(\log (K)) .
$$

It follows that $\bar{w}$ solves (2.18). To show that the right hand side in $(2.18) f \in\left(H_{\nu}^{1}(\mathbb{R})\right)^{*}$ for all $\nu>0$, note that for arbitrary $v \in H_{\nu}^{1}(\mathbb{R})$

$$
\left|\langle f, v\rangle_{\left(H_{\nu}^{1}(\mathbb{R})\right)^{*} \times H_{\nu}^{1}(\mathbb{R})}\right|=\left|\frac{\sigma^{2}}{2} K v(\log (K)) \mathrm{e}^{2 \nu|\log (K)|}\right| \leq C(\nu, \sigma, K)|v(\log (K))| \leq C(\nu, \sigma, K)\|v\|_{H_{\nu}^{1}(\mathbb{R})} .
$$

Multiplying (2.18) by the test function $v(x) \mathrm{e}^{2 \nu|x|}$, with $v \in C_{0}^{\infty}(\mathbb{R})$ we obtain

$$
\begin{aligned}
\frac{\mathrm{d}}{\mathrm{d} \tau}(\bar{w}(\tau, \cdot), v)_{L_{\nu}^{2}(\mathbb{R}) \times L_{\nu}^{2}(\mathbb{R})}+a^{\nu}(\bar{w}, v) & =\langle f, v\rangle_{\left(H_{\nu}^{1}(\mathbb{R})\right)^{*} \times H_{\nu}^{1}(\mathbb{R})} \quad \forall v \in C_{0}^{\infty}(\mathbb{R}) \\
\bar{w}(0, x) & =0 .
\end{aligned}
$$


By Proposition 2.1 and Theorem 2.3, there exists a unique $\bar{w} \in L^{2}\left(0, T ; H_{\nu}^{1}(\mathbb{R})\right) \cap H^{1}\left(0, T ;\left(H_{\nu}^{1}(\mathbb{R})\right)^{*}\right)$ solution to $(2.20)$. It satisfies $(2.15)$ with $V=H_{\nu}^{1}(\mathbb{R})$ for $\nu>0$ which implies exponential decay of $|\bar{w}|$ as $|x| \rightarrow \infty$.

The case $r \neq 0$ is reduced to $r=0$ by transformation to "transformed" variables

$$
w(\tau, x)=\mathrm{e}^{-r \tau} \check{w}(\tau, x+r \tau)
$$

which reduces the original problem for $w$ to a BS equation for $\check{w}$ with $r=0$ :

$$
\frac{\partial \breve{w}}{\partial \tau}-\frac{\sigma^{2}}{2} \frac{\partial^{2} \breve{w}}{\partial x^{2}}+\frac{\sigma^{2}}{2} \frac{\partial \breve{w}}{\partial x}=0, \quad \check{w}(0, x)=h(x) .
$$

We shall use (2.21) in several places and refer to quantities like $\check{w}$ as "transformed" variables, without indication by ${ }^{2}$.

\section{Pricing European vanillas on LÉvy Driven assets}

\subsection{Lévy processes}

Let $\left(\Omega, \mathcal{F},\left(\mathcal{F}_{t}\right)_{0 \leq t<\infty}, \mathbf{P}\right)$ be a filtered probability space. An adapted process $\left(X_{t}\right)_{t \geq 0}$ is called a Lévy process if

(1) (independent increments) for every $s, t \geq 0, X_{t+s}-X_{s}$ is independent of $X_{s}$;

(2) $X_{0}=0 \mathbf{P}$ - a.s;

(3) (temporal homogeneity or stationary increments property) the distribution of $X_{t+s}-X_{s}$ does not depend of $s$;

(4) it is stochastically continuous, i.e., $\lim _{t \rightarrow 0^{+}} \mathbf{P}\left[\left|X_{t}\right|>\varepsilon\right]=0$ for any $\varepsilon>0$.

Since any process $X_{t}$ satisfying (1)-(4) has a cadlag modification we will always assume $X_{t}$ to be cadlag. The Lévy-Khintchine formula describes explicitly a Lévy process in terms of its Fourier transform $E_{\mathbb{Q}}\left[\mathrm{e}^{-i u X_{t}}\right]$ under a chosen equivalent martingale measure $\mathbb{Q}$ :

$$
E_{\mathbb{Q}}\left[\mathrm{e}^{-i u X_{t}}\right]=\mathrm{e}^{-t \psi(u)}
$$

for some function $\psi$ called the Lévy exponent of $X$. By the Lévy-Khintchine formula,

$$
\psi(u)=\frac{\sigma^{2}}{2} u^{2}+i \alpha u+\int_{|x|<1}\left(1-\mathrm{e}^{-i u x}-i u x\right) \nu_{\mathbb{Q}}(\mathrm{d} x)+\int_{|x| \geq 1}\left(1-\mathrm{e}^{-i u x}\right) \nu_{\mathbb{Q}}(\mathrm{d} x)
$$

so that a Lévy process is characterized by the Lévy triple $\sigma, \alpha \in \mathbb{R}$ and the Lévy-measure $\nu_{\mathbb{Q}}$ on $\mathbb{R} \backslash\{0\}$ satisfying

$$
\int \min \left(1, x^{2}\right) \nu_{\mathbb{Q}}(\mathrm{d} x)<\infty
$$

The characteristic exponent $\psi$ turns out to be the symbol of the pseudo-differential operator $\mathcal{A}$ which is the infinitesimal generator of the transition semi-group of $X_{t}$ under the equivalent martingale measure $\mathbb{Q}$. We assume here that the equivalent martingale measure $\mathbb{Q}$ has been chosen by some procedure, we refer to $[13,16,17,19]$ and the references therein for various results in this direction.

\subsection{Price processes}

In Lévy markets, log returns of the risky assets are modelled by a Lévy process. The spot price $S_{t}$ of the risky asset is

$$
S_{t}=S_{0} \mathrm{e}^{\left(r-\sigma^{2} / 2+c\right) t+X_{t}}
$$


where $X_{t}$ is a Lévy process. By (3.2) and (3.3), $X_{t}=\sigma B_{t}+Y_{t}$, with $B_{t}$ a brownian motion and $Y_{t}$ a quadratic pure jump Lévy process independent of $B_{t}$. The parameter $c$ in (3.4) is chosen so that the mean rate of return on the asset is risk-neutrally $r$, i.e. $\mathrm{e}^{-c t}=E_{\mathbb{Q}}\left[\mathrm{e}^{Y_{t}}\right]$.

Let $\mu(\mathrm{d} x, \mathrm{~d} t)$ denote the integer valued random measure (the jump measure) that counts the number of jumps of $Y_{t}$ in space-time. By Ito's formula (e.g. Th. 4.57 in [20]), $S_{t}$ solves the following stochastic differential equation

$$
\mathrm{d} S_{t}=S_{t_{-}} \mathrm{d} X_{t}+S_{t_{-}} \int_{\mathbb{R}}\left(\mathrm{e}^{y}-1-y\right) \mu(\mathrm{d} y, \mathrm{~d} t)+(r+c) \mathrm{d} t
$$

By stationarity of Lévy processes, the compensator of the measure $\mu(\mathrm{d} x, \mathrm{~d} t)$ has the form $\nu_{\mathbb{Q}}(\mathrm{d} x) \times \mathrm{d} t$, with $\mathrm{d} t$ being the Lebesgue measure.

In the following we will assume that the Lévy measure $\nu_{\mathbb{Q}}$ has a density $k_{\mathbb{Q}}$, i.e., $\nu_{\mathbb{Q}}(\mathrm{d} z)=k_{\mathbb{Q}}(z) \mathrm{d} z$ and we will drop the subscript $\mathbb{Q}$. The Lévy density $k(z)$ describes the activity of jumps of size $z$ in $X_{t}$. Lévy processes are said to be of finite activity, if $k(z)$ is integrable near $z=0$, otherwise of infinite activity.

In our analysis, we use some or all of the following assumptions on the Lévy density $k$.

(A1) (activity of small jumps) the characteristic function $\psi_{0}(u)$ of the pure jump part $Y_{t}$ of the Lévy process $X_{t}$ satisfies: there exist constants $c_{1}, C_{+}>0$ and $Y<2$ such that

$$
\left|\psi_{0}(u)-i c_{1} u\right| \leq C_{+}\left(1+|u|^{2}\right)^{Y / 2} \quad \forall u \in \mathbb{R} .
$$

(A2) (semiheavy tails) there are constants $C>0, G>0$ and $M>1$ such that

$$
\forall|z|>1: \quad k(z) \leq C \begin{cases}\mathrm{e}^{-G|z|} \text { if } z<0 \\ \mathrm{e}^{-M|z|} \text { if } z>0\end{cases}
$$

(A3) (smoothness)

$$
\forall \alpha \in \mathbb{N}_{0} \exists C(\alpha): \quad \forall z \neq 0:\left|\partial_{z}^{\alpha} k(z)\right| \leq C(\alpha)|z|^{-(1+Y+\alpha)_{+}} .
$$

If $\sigma=0$ we assume $0<Y<2$ and in addition.

(A4) (boundedness from below of $k(z)$ ): there is $C_{-}>0$ such that

$$
\forall 0<|z|<1: \quad \frac{1}{2}(k(-z)+k(z)) \geq \frac{C_{-}}{|z|^{1+Y}} .
$$

Remark 3.1. (i) By (3.4), (3.1) and (3.2) and by $E_{\mathbb{Q}}\left[S_{t}\right]<\infty$ we obtain that $E_{\mathbb{Q}}\left[\mathrm{e}^{X_{t}}\right]=\mathrm{e}^{-t \psi(i)}<\infty$, with $\psi$ being the Lévy exponent in (3.2). As a consequence, the Lévy density $k$ has to satisfy both the integrability condition (3.3) and $\int_{|z|>1} \mathrm{e}^{z} k(z) \mathrm{d} z<\infty$. This holds for $k$ satisfying (A1) and (A2), due to $Y<2$ and $M>1$ which we shall assume throughout.

(ii) Assumption (A2) implies in particular that $X_{t}$ has finite moments of all orders.

(iii) We will require (A3) in particular in the analysis of the wavelet compression of the moment matrix of $k(z)$; it is, however, required only for a finite range of $\alpha$.

(iv) A Lévy process $X_{t}$ with $\sigma=0$ in the Lévy triple is called pure jump process. If $X_{t}$ is a pure jump process, we assume that it is of infinite activity, i.e.

$$
k(z) \text { satisfies }(\mathrm{A} 1)-(\mathrm{A} 4) \text { with } 0<Y<2 \text { if } \sigma=0 .
$$

(v) If the Lévy process is of finite activity, we assume that $\sigma>0$ and that $k(z)$ satisfies (A1) $-($ A3) with $Y<0$.

\subsection{Examples of Lévy processes}

All price processes used in Lévy market models known to us have densities which satisfy (A1)-(A3). For example, the generalized hyperbolic motions $[3,18,35]$ satisfy (A1) with $Y=1$. Further specific examples of Lévy processes follow; for their explicit characteristic functions we refer to [40]. 


\subsubsection{Merton model}

In the classical Merton model [31], the spot price $S_{t}$ is modelled by a drifted brownian motion with finitely many jumps, i.e. $X_{t}=\sigma B_{t}+\sum_{i=1}^{N_{t}} Y_{i}$ where $\left\{Y_{i}\right\}$ are independent, identically distributed random variables with distribution function $f(z)$ and where $\left\{N_{t}\right\}$ is a Poisson process with intensity $\lambda$. The Lévy measure is $\nu(\mathrm{d} z)=k(z) \mathrm{d} z$ with $k(z)=\lambda f(z)$.

Merton assumed a normal distribution with mean $\mu_{M}$ and standard deviation $\sigma_{M}$ where $f_{M}(z)=\left(\sqrt{2 \pi} \sigma_{M}\right)^{-1} \exp \left(-\left(z-\mu_{M}\right)^{2} /\left(2 \sigma_{M}^{2}\right)\right)$. With this density, Merton's model is a finite intensity Lévy process which satisfies (A1)-(A3) with $Y=-\infty$. To accommodate asymmetric distributions of positive and negative jumps in returns, Kou [24] proposed $f_{\text {Kou }}(z)=p_{+} M \exp (-M z) \chi_{\mathbb{R}_{+}}(z)+p_{-} G \exp (G z) \chi_{\mathbb{R}_{-}}(z), p_{+}+p_{-}=1$. Then $X_{t}$ is a finite activity Lévy process with $k$ satisfying (A1)-(A3) for $Y=-1$.

\subsubsection{CGMY process}

The CGMY process [12] assumes an infinitely divisible four parameter distribution of the log-returns that allows the resulting Lévy process to have both finite or infinite activity and finite or infinite variation. The Lévy density of the CGMY process is given by ([12])

$$
k_{C G M Y}(z)=C\left\{\begin{array}{l}
\frac{\mathrm{e}^{-G|z|}}{|z|^{1+Y}} \text { if } z<0 \\
\frac{\mathrm{e}^{-M|z|}}{|z|^{1+Y}} \text { if } z>0
\end{array}\right.
$$

where $C>0, G, M \geq 0$ and $Y<2$. The case $Y=0$ is the special case of the variance gamma process. The density (3.11) satisfies (A1)-(A4).

\subsubsection{Normal inverse gaussian process}

With $\left(B^{1}, B^{2}\right)$ being a bivariate brownian motion starting at $(\mu, 0)$ and with constant drift vector $(\beta, \gamma)$, let $\tau$ denote the time at which the second component $B^{2}$ hits the line $B^{2}=\delta>0$ for the first time. Then, with $\alpha=\sqrt{\beta^{2}+\gamma^{2}}$, the law of $B^{1}(\tau)$ is $N I G(\alpha, \beta, \mu, \delta)[4]$. The three-parameter Lévy density of the NIG Lévy process takes the form

$$
k_{N I G}(z)=\frac{1}{\pi} \delta \alpha \frac{1}{|z|} K_{1}(\alpha|z|) \mathrm{e}^{\beta z}
$$

where $K_{1}$ is the modified Bessel function of the third kind. It satisfies (A1)-(A4) with $Y=0$.

\subsubsection{Meixner process}

The Meixner process was proposed in [40]. It is an infinite activity pure jump process with a three parameter Lévy density given by

$$
k_{\text {Meixner }}(z)=\delta \frac{\exp (\beta z / \alpha)}{z \sinh (\pi z / \alpha)} .
$$

It easily verified that $k_{\text {Meixner }}(z)$ satisfies (A1)-(A3) with $Y=1$ and suitable $G(\alpha, \beta), M(\alpha, \beta)$ if $\alpha, \delta>0$ and $|\beta|<\pi$.

\subsection{Partial integro-differential equation (PIDE)}

Let $f\left(t, S_{t}\right)$ denote the price at time $t$ of a contingent claim on the asset $S_{t}$ in (3.4). We consider here an European call option, i.e. $f\left(T, S_{T}\right)=g\left(S_{T}\right):=\left(S_{T}-K\right)_{+}$, with strike price $K$ and maturity $T$. The price $f\left(t, S_{t}\right)$ can be calculated for all dates $t<T$ by taking conditional expectations. Assuming that the savings account process is given by $S_{t}^{0}=\mathrm{e}^{r t}$, the process $\mathrm{e}^{-r t} S_{t}$ is a martingale under $\mathbb{Q}$, since $\mathbb{Q}$ is assumed to be the risk-neutral measure. The same holds true for the value process $f\left(t, S_{t}\right)$ of the option, therefore

$$
f\left(t, S_{t}\right)=E_{\mathbb{Q}}\left[\mathrm{e}^{r(t-T)} g\left(S_{T}\right) \mid \mathcal{F}_{t}\right] .
$$


The key to fast deterministic valuation of $f\left(t, S_{t}\right)$ is the following result $(e . g .[32,37])$. It characterizes $f\left(t, S_{t}\right)$ with sufficient regularity as solution of a deterministic partial integro-differential equation (PIDE).

Unless explicitly stated otherwise, we assume in the following that $X_{t}$ has a non-zero diffusion component, i.e. $\sigma>0$. We also change to $\operatorname{logarithmic}$ price $x=\log (S) \in \mathbb{R}$ and time to maturity $\tau=T-t$.

Theorem 3.2. Assume $u(\tau, x) \in C^{1,2}\left((0, T) \times \mathbb{R}_{+}\right) \cap C^{0}\left([0, T] \times \mathbb{R}_{+}\right)$solves the PIDE

$$
\frac{\partial u}{\partial \tau}-\frac{\sigma^{2}}{2} \frac{\partial^{2} u}{\partial x^{2}}+\left(\frac{\sigma^{2}}{2}-r+c_{\exp }\right) \frac{\partial u}{\partial x}+A[u]+r u=0 \quad \text { in }(0, T) \times \mathbb{R}
$$

where A denotes the integro-differential operator

$$
A[\varphi](x):=-\int_{\mathbb{R}}\left\{\varphi(x+y)-\varphi(x)-y \varphi^{\prime}(x) \chi_{\{|y| \leq 1\}}\right\} k(y) \mathrm{d} y
$$

and $c_{\exp } \in \mathbb{R}$ is given by

$$
c_{\exp }:=-\mathrm{e}^{-x} A[\exp (\cdot)](x)=\int_{\mathbb{R}}\left\{\mathrm{e}^{y}-1-y \chi_{\{|y| \leq 1\}}\right\} k(y) \mathrm{d} y
$$

together with the initial condition

$$
u(0, x)=h(x)
$$

where $h(x):=g\left(\mathrm{e}^{x}\right)$. Then $f(t, S):=u(T-t, \log (S))$ satisfies

$$
f\left(t, S_{t}\right)=E_{\mathbb{Q}}\left[\mathrm{e}^{r(t-T)} g\left(S_{T}\right) \mid \mathcal{F}_{t}\right] .
$$

Conversely, if $f(t, S)$ in (3.18) is sufficiently regular, then $u(\tau, x):=f\left(T-\tau, \mathrm{e}^{x}\right)$ is solution of (3.14), (3.17).

For the numerical solution below, it will be important to have information on the spectrum of the integral operator $A$.

Remark 3.3. $A+A^{*} \geq 0$. More precisely, for all $\varphi, \psi \in H^{1}(\mathbb{R})$ there holds

$$
(A[\varphi], \psi)_{L^{2}(\mathbb{R})}+(A[\psi], \varphi)_{L^{2}(\mathbb{R})}=\int_{\mathbb{R}} \int_{\mathbb{R}}(\varphi(x+y)-\varphi(x))(\psi(x+y)-\psi(x)) k(y) \mathrm{d} y \mathrm{~d} x .
$$

\subsection{Variational formulation}

Our pricing methodology is based on the numerical solution of the PIDE (3.14). Numerical solution of PIDEs for European vanillas by characteristic functions and FFT techniques has been advocated in [11]. Our solution algorithm aims at American put and Barrier options (see [30]). It will be based on a variational formulation of the PIDE which we now give. As in the Black-Scholes setting, the variational formulation of the PIDE (3.14) will be based on weighted Sobolev spaces allowing exponential growth of the solution at $\infty$.

\subsubsection{Weighted spaces}

Let $\eta \in L_{\text {loc }}^{1}(\mathbb{R}), \eta^{\prime} \in L^{\infty}(\mathbb{R})$. We denote by $H_{\eta}^{1}(\mathbb{R})$ the weighted Sobolev space given by

$$
H_{\eta}^{1}(\mathbb{R}):=\left\{\varphi \in L_{\mathrm{loc}}^{1}(\mathbb{R}): \mathrm{e}^{\eta} \varphi, \mathrm{e}^{\eta} \varphi^{\prime} \in L^{2}(\mathbb{R})\right\}
$$

We observe that the pay-off function $h$ in $(3.17)$ satisfies $h \in H_{-\zeta}^{1}(\mathbb{R})$ for all $\zeta$ of the form

$$
\zeta(x)= \begin{cases}\mu_{1}|x| & \text { if } x<0 \\ \mu_{2}|x| & \text { if } x>0\end{cases}
$$


for all $\mu_{1}>0$ and $\mu_{2}>1$. We will denote by $\mathcal{A}$ the spatial operator in (3.14) given by

$$
\mathcal{A}[\varphi](x):=-\frac{\sigma^{2}}{2} \frac{\mathrm{d}^{2} \varphi}{\mathrm{d} x^{2}}(x)+\left(\frac{\sigma^{2}}{2}-r+c_{\exp }\right) \frac{\mathrm{d} \varphi}{\mathrm{d} x}(x)+r \varphi+A[\varphi](x) .
$$

For $\varphi, \psi \in C_{0}^{\infty}(\mathbb{R})$ we associate with $\mathcal{A}$ the bilinear form

$$
a^{\eta}(\varphi, \psi):=\int_{\mathbb{R}} \mathcal{A}[\varphi](x) \psi(x) \mathrm{e}^{2 \eta(x)} \mathrm{d} x .
$$

In the case $\eta=0$, we write $a(\varphi, \psi)$ in place of $a^{0}(\varphi, \psi)$, i.e.

$$
a(\varphi, \psi)=\int_{\mathbb{R}}\left(\frac{\sigma^{2}}{2} \varphi^{\prime} \psi^{\prime}+\left[\frac{\sigma^{2}}{2}-r+c_{\exp }\right] \varphi^{\prime} \psi+r \varphi \psi\right) \mathrm{d} x+\langle A[\varphi], \psi\rangle_{H^{1}(\mathbb{R})^{*} \times H^{1}(\mathbb{R})} .
$$

For certain weighting functions $\eta \in L_{\mathrm{loc}}^{1}(\mathbb{R}), \eta^{\prime} \in L^{\infty}(\mathbb{R})$, the bilinear form $a^{\eta}(\cdot, \cdot)$ can be extended continuously to $H_{\eta}^{1}(\mathbb{R}) \times H_{\eta}^{1}(\mathbb{R})$. Moreover, under certain conditions on $\eta$ this bilinear form is, up to a $L_{\eta}^{2}$-scalar product, coercive on $H_{\eta}^{1}(\mathbb{R}) \times H_{\eta}^{1}(\mathbb{R})$ in the sense that the following analogue of Proposition 2.1 holds.

Theorem 3.4. Let $\eta \in L_{\text {loc }}^{1}(\mathbb{R})$ such that $\eta^{\prime} \in L^{\infty}(\mathbb{R})$ and assume that $r=0$ in (3.21), (3.22).

(1) If

and

$$
\eta(x+\theta y)-\eta(x) \leq \eta(y) \quad \forall x, y \in \mathbb{R} \quad \forall \theta \in[0,1]
$$

$$
C(\eta):=\int_{\mathbb{R}} \mathrm{e}^{\eta(y)}|y| \chi_{\{|y| \leq 1\}}(y) k(y) \mathrm{d} y<+\infty
$$

hold, there exist $\alpha_{\eta}, \beta_{\eta}>0$ and $C_{\eta}>0$ such that

$$
\begin{aligned}
\left|a^{-\eta}(\varphi, \psi)\right| & \leq C_{\eta}\|\varphi\|_{H_{-\eta}^{1}(\mathbb{R})}\|\psi\|_{H_{-\eta}^{1}(\mathbb{R})} \quad \forall \varphi, \psi \in H_{-\eta}^{1}(\mathbb{R}) \\
a^{-\eta}(\varphi, \varphi) & \geq \alpha_{\eta}\|\varphi\|_{H_{-\eta}^{1}(\mathbb{R})}^{2}-\beta_{\eta}\|\varphi\|_{L_{-\eta}^{2}(\mathbb{R})}^{2} \quad \forall \varphi \in H_{-\eta}^{1}(\mathbb{R}) .
\end{aligned}
$$

(2) If $\eta$ is such that

$$
-\eta(x+\theta y)+\eta(x) \leq \eta(-y) \quad \forall x, y \in \mathbb{R} \quad \forall \theta \in[0,1]
$$

and

$$
\tilde{C}(-\eta):=\int_{\mathbb{R}} \mathrm{e}^{\eta(-y)}|y| \chi_{\{|y| \geq 1\}}(y) k(y) \mathrm{d} y<+\infty
$$

hold, there exist $\alpha_{\eta}^{\prime}, \beta_{\eta}^{\prime}>0$ and $C_{\eta}^{\prime}>0$ such that

$$
\begin{aligned}
\left|a^{\eta}(\varphi, \psi)\right| & \leq C_{\eta}^{\prime}\|\varphi\|_{H_{\eta}^{1}(\mathbb{R})}\|\psi\|_{H_{\eta}^{1}(\mathbb{R})} \quad \forall \varphi, \psi \in H_{\eta}^{1}(\mathbb{R}) \\
a^{\eta}(\varphi, \varphi) & \geq \alpha_{\eta}^{\prime}\|\varphi\|_{H_{\eta}^{1}(\mathbb{R})}^{2}-\beta_{\eta}^{\prime}\|\varphi\|_{L_{\eta}^{2}(\mathbb{R})}^{2} \quad \forall \varphi \in H_{\eta}^{1}(\mathbb{R}) .
\end{aligned}
$$

The proof of this theorem is given in Appendix A.

\subsubsection{Reduction to homogeneous initial condition}

We return to (3.14)-(3.17). Since $h \in H_{-\zeta}^{1}(\mathbb{R})$ for all $\zeta$ as in (3.20), we cast (3.14)-(3.17) in the following weak form: find $u \in L^{2}\left(0, T ; H_{-\zeta}^{1}(\mathbb{R})\right) \cap H^{1}\left(0, T ;\left(H_{-\zeta}^{1}(\mathbb{R})\right)^{*}\right)$ such that

$$
\begin{gathered}
\frac{\mathrm{d}}{\mathrm{d} \tau}(u(\tau, \cdot), v)_{L_{-\zeta}^{2}(\mathbb{R})}+a^{-\zeta}(u(\tau, \cdot), v)=0 \quad \forall v \in H_{-\zeta}^{1}(\mathbb{R}), \\
u(0, \cdot)=h \quad \text { in } H_{-\zeta}^{1}(\mathbb{R}) .
\end{gathered}
$$


By Theorem 3.4, Item (1), and Theorem 2.3, applied in the triple $X=H_{-\zeta}^{1}(\mathbb{R}) \hookrightarrow L_{-\zeta}^{2}(\mathbb{R}) \cong\left(L_{-\zeta}^{2}(\mathbb{R})\right)^{*} \hookrightarrow X^{*}$, (3.28) admits a unique weak solution $u \in L^{2}\left(0, T ; H_{-\zeta}^{1}(\mathbb{R})\right) \cap H^{1}\left(0, T ;\left(H_{-\zeta}^{1}(\mathbb{R})\right)^{*}\right)$.

For numerical computations we compute the excess to "transformed" payoff on a bounded domain with homogeneous initial and artificial zero boundary conditions. We transform to $r=0$ by (2.21) and remove inhomogeneous initial condition by a particular solution. To this end, we analyze the image of the pay-off function $h(x)$ under the operator $\mathcal{A}$ and write the operator $\mathcal{A}$ as

$$
\mathcal{A}=-\frac{\sigma^{2}}{2} \frac{\mathrm{d}^{2}}{\mathrm{~d} x^{2}}+\left(\frac{\sigma^{2}}{2}-r\right) \frac{\mathrm{d}}{\mathrm{d} x}+r+\hat{A}
$$

with

$$
\hat{A}[\phi](x):=-\int_{\mathbb{R}}\left[\phi(x+z)-\phi(x)-z \phi^{\prime}(x) \chi_{\{|z| \leq 1\}}\right] k(z) \mathrm{d} z+c_{\exp } \phi^{\prime}(x),
$$

with density function $k(z)$ satisfying the integrability conditions (3.3) and $\int_{|z| \geq 1} \mathrm{e}^{z} k(z) \mathrm{d} z<\infty$, see also Remark 3.1, (i). The constant $c_{\exp }$ is chosen as in (3.16) so that $\hat{A}\left[\mathrm{e}^{x}\right]=0$ and, by (2.21), we may and will assume $r=0$ in what follows.

The operator $\hat{A}$ in (3.29) satisfies a strong pseudo-local property: it preserves singular support and exponential decay at $\infty$. We exemplify this here for a European call.

Theorem 3.5. Assume that the Lévy measure of $X_{t}$ has the form $\nu(\mathrm{d} z)=k(z) \mathrm{d} z$ with $k(z)$ satisfying (A1), (A2). Let $h$ be the payoff for a European call, $h(x)=\left(\mathrm{e}^{x}-K\right)+$ and set $\psi:=-\hat{A}[h]$. Then $\psi \in C^{\infty}(\mathbb{R} \backslash\{\log (K)\}) \cap$ $L_{\text {loc }}^{1}(\mathbb{R})$ and $\psi$ decays exponentially at $\pm \infty$ : there exist $C_{1}, C_{2}>0$ such that $0 \leq \psi(x) \leq C_{1} \mathrm{e}^{-G x}$ for $x>0$ sufficiently large and $0 \leq \psi(x) \leq C_{2} \mathrm{e}^{M x}$ for $x<0$ and $|x|$ sufficiently large. Hence, $\psi \in\left(H_{\eta}^{1}(\mathbb{R})\right)^{*}$ for all $\eta \geq 0$ satisfying (3.26), (3.27) and, in particular, for $\eta=0$.

Proof. Let $x>\log (K)$. Then there holds

$$
\begin{aligned}
\psi(x)= & \left.\int_{\mathbb{R}}\left[\left(\mathrm{e}^{x+z}-K\right)_{+}-\left(\mathrm{e}^{x}-K\right)_{+}-z\left(\left(\mathrm{e}^{x}-K\right)_{+}\right)^{\prime} \chi_{\{|z| \leq 1\}}\right)\right] k(z) \mathrm{d} z-c_{\exp }\left(\left(\mathrm{e}^{x}-K\right)_{+}\right)^{\prime} \\
= & \int_{-\infty}^{\log (K)-x}\left[0-\left(\mathrm{e}^{x}-K\right)-z \mathrm{e}^{x} \chi_{\{|z| \leq 1\}}\right] k(z) \mathrm{d} z \\
& +\int_{\log (K)-x}^{\infty}\left[\mathrm{e}^{x+z}-\mathrm{e}^{x}-z \mathrm{e}^{x} \chi_{\{|z| \leq 1\}}\right] k(z) \mathrm{d} z-c_{\exp } \mathrm{e}^{x} .
\end{aligned}
$$

By the choice of $c_{\exp }$ in $(3.16)$ we obtain that

$$
\begin{aligned}
\psi(x) & =\int_{-\infty}^{\log (K)-x}\left[K-\mathrm{e}^{x}-z \mathrm{e}^{x} \chi_{\{|z| \leq 1\}}\right] k(z) \mathrm{d} z-\int_{-\infty}^{\log (K)-x}\left[\mathrm{e}^{x+z}-\mathrm{e}^{x}-z \mathrm{e}^{x} \chi_{\{|z| \leq 1\}}\right] k(z) \mathrm{d} z \\
& =\int_{-\infty}^{\log (K)-x}\left(K-\mathrm{e}^{x+z}\right) k(z) \mathrm{d} z, \quad \log (K)-x<0 .
\end{aligned}
$$

Analogously we obtain that

$$
\psi(x)=\int_{\log (K)-x}^{\infty}\left(\mathrm{e}^{x+z}-K\right) k(z) \mathrm{d} z, \quad \log (K)-x>0 .
$$


With $k$ satisfying (A1), (A2) and with

$$
\psi(x)=\left\{\begin{array}{l}
\int_{-\infty}^{\log (K)-x}\left(K-\mathrm{e}^{x+z}\right) k(z) \mathrm{d} z, x>\log (K) \\
\int_{\log (K)-x}^{\infty}\left(\mathrm{e}^{x+z}-K\right) k(z) \mathrm{d} z, \quad x<\log (K),
\end{array}\right.
$$

we obtain that $\psi \in C^{\infty}(\mathbb{R} \backslash\{\log (K)\}$, i.e. $\operatorname{sing} \operatorname{supp} \psi=\{\log (K)\}$.

We claim that $|\log (K)-x|^{p} \psi(x) \in L^{\infty}(\mathbb{R})$ for $p=Y-1$. Indeed, with $C_{*}:=\lim _{z \rightarrow 0^{-}}|z|^{1+Y} k(z)$

$$
\begin{aligned}
\lim _{x \downarrow \log (K)}|\log (K)-x|^{p} \psi(x) & =\lim _{\varepsilon \downarrow 0} \varepsilon^{p} \int_{-\infty}^{-\varepsilon}\left(K-K \mathrm{e}^{\varepsilon} \mathrm{e}^{z}\right) k(z) \mathrm{d} z \\
& =\lim _{\varepsilon \downarrow 0} K \varepsilon^{p} \int_{\varepsilon}^{\infty}\left(1-\mathrm{e}^{\varepsilon} \mathrm{e}^{-z}\right) k(-z) \mathrm{d} z \\
& =\lim _{\varepsilon \downarrow 0} \frac{K}{p} \varepsilon^{p+1} \int_{\varepsilon}^{\infty} \mathrm{e}^{-z} k(-z) \mathrm{d} z \\
& =K \frac{C_{*}}{p(p+1)} \lim _{\varepsilon \downarrow 0} \varepsilon^{p+2-Y-1}=K \frac{C_{*}}{p(p+1)} .
\end{aligned}
$$

Since $Y<2, p=Y-1<1$, therefore $\psi \in C^{\infty}(\mathbb{R} \backslash\{\log (K)\}) \cap L_{\text {loc }}^{1}(\mathbb{R})$. Moreover, $\psi$ decays exponentially at $\pm \infty$. More precisely, for $x>\max \{\log (K)+1,0\}, \psi(x) \leq C K^{G+1} \mathrm{e}^{-G x}$, and for $x \leq \min \{\log (K)-1,0\}$, $\psi(x) \leq C K^{1-M} \mathrm{e}^{M x}$. Consequently, $\psi \in\left(H_{\eta}^{1}(\mathbb{R})\right)^{*}$ for all $\eta \geq 0$ satisfying (3.26) and (3.27), in particular, for $\eta=0$.

Positive weighting exponents $\eta$ satisfying (3.26), (3.27) are, e.g.,

$$
\eta(x)= \begin{cases}\eta_{-}|x|, & x<0 \\ \eta_{+}|x|, & x>0\end{cases}
$$

for $0<\eta_{-}<M$ and $0<\eta_{+}<G$ with $G, M$ as in (A2).

Proposition 3.6. Assume $r=0$ in (3.21). Then, for $h$ as in Theorem $3.5,-\mathcal{A}[h] \in\left(H_{\eta}^{1}(\mathbb{R})\right)^{*}$ for all $\eta$ as in (3.26), (3.27) and, in particular, for $\eta=0$.

Proof. By the transformation (2.21), it is sufficient to consider $\mathcal{A}=-\frac{\sigma^{2}}{2} \frac{\mathrm{d}^{2}}{\mathrm{~d} x^{2}}+\frac{\sigma^{2}}{2} \frac{\mathrm{d}}{\mathrm{d} x}+\hat{A}$. Therefore, $-\mathcal{A}[h]=$ $\frac{\sigma^{2}}{2} K \delta_{\log (K)}-\hat{A}[h]$. By Theorem 3.5, $-\mathcal{A}[h] \in\left(H_{\eta}^{1}(\mathbb{R})\right)^{*}$ for all $\eta$ as in (3.26), (3.27) and, in particular, for $\eta=0$.

Let $u$ denote the solution of the parabolic evolution problem (3.14)-(3.17) and denote by $\mathcal{A}$ the spatial operator given by (3.21) with $r=0$. By Proposition 3.6, $-\mathcal{A}[h] \in\left(H_{\eta}^{1}(\mathbb{R})\right)^{*}$ for all $\eta$ satisfying (3.27) and (3.26) and the excess to "transformed" payoff $U:=u-\mathrm{e}^{-r t} h(\cdot+r t)$ solves

$$
\begin{aligned}
\frac{\partial U}{\partial \tau}+\mathcal{A}[U] & =f:=-\mathcal{A}[h] \quad \text { in } \quad(0, T) \times \mathbb{R}, \\
\left.U\right|_{\tau=0} & =0 \quad \text { in } \mathbb{R}
\end{aligned}
$$

or, in variational form: find $U \in L^{2}\left((0, T) ; H_{\eta}^{1}(\mathbb{R})\right) \cap H^{1}\left(0, T ;\left(H_{\eta}^{1}(\mathbb{R})\right)^{*}\right)$ such that

$$
\begin{aligned}
\left(\frac{\mathrm{d}}{\mathrm{d} \tau} U(\tau, \cdot), v\right)_{L_{\eta}^{2}(\mathbb{R})}+a^{\eta}(U(\tau, \cdot), v) & =\langle f, v\rangle_{\left(H_{\eta}^{1}(\mathbb{R})\right)^{*} \times H_{\eta}^{1}(\mathbb{R})} \quad \forall v \in H_{\eta}^{1}(\mathbb{R}) \\
\left.U\right|_{\tau=0} & =0 .
\end{aligned}
$$


With $V:=H_{\eta}^{1}(\mathbb{R})$ and $H:=L_{\eta}^{2}(\mathbb{R})$ we have $V \hookrightarrow H \cong H^{*} \hookrightarrow V^{*}$ with dense embeddings. By Theorems 3.4 and 2.3, applied to $\mathcal{A} \in \mathcal{L}\left(V, V^{*}\right), V=H_{\eta}^{1}(\mathbb{R})$, given $f \in\left(H_{\eta}^{1}(\mathbb{R})\right)^{*}$, there exists a unique weak solution $U \in L^{2}\left(0, T ; H_{\eta}^{1}(\mathbb{R})\right) \cap H^{1}\left(0, T ;\left(H_{\eta}^{1}(\mathbb{R})\right)^{*}\right)$ of $(3.32)$ and (3.33). Indeed, by Theorem 3.4, item 2., there exists $\lambda>0$ such that the shifted operator $\mathcal{A}+\lambda$. id induces a coercive bilinear form on $H_{\eta}^{1}(\mathbb{R}) \times H_{\eta}^{1}(\mathbb{R})$.

We prove an a priori estimate for the weak solution $U$ of (3.32) and (3.33). To this end, let us denote by $T^{\mathcal{A}+\lambda \cdot \text { id }}(\cdot)$ the analytic semi-group induced by the operator $\mathcal{A}+\lambda \cdot \mathrm{id}$ in $\left(H_{\eta}^{1}(\mathbb{R})\right)^{*}$ and let $f:=-\mathcal{A}[h]$. Then $U$ admits the Duhamel's representation in $\left(H_{\eta}^{1}(\mathbb{R})\right)^{*}$, see e.g. [2], Proposition III.1.3.1,

$$
U(\tau)=\int_{0}^{\tau} T^{\mathcal{A}+\lambda \cdot \mathrm{id}}(s)[f] \mathrm{e}^{-\lambda s} \mathrm{~d} s .
$$

Recall that by Theorem 3.5 and Proposition 3.6, $f=\sigma^{2} / 2 K \delta_{\log (K)}+\psi$, with $\psi \in C^{\infty}(\mathbb{R} \backslash \log (K)) \cap L_{\operatorname{loc}}^{1}(\mathbb{R})$ decaying exponentially at $\pm \infty$. Therefore, $f \in\left(H_{\eta}^{1 / 2+\varepsilon}(\mathbb{R})\right)^{*}$ for all $\varepsilon>0$.

We denote by $V_{\theta}:=\left[V^{*}, V\right]_{\theta, 2}$ the interpolation space for $0 \leq \theta \leq 1$ between $V^{*}$ and $V\left(V_{0}=V^{*}\right.$ and $V_{1}=V$ ). Then there exists $\theta>0$ such that $f \in V_{\theta}$ and there exist $C, d>0$ such that for all $t>0$ (see [39], Th. 1)

$$
\left\|\left(T^{\mathcal{A}+\lambda \cdot \mathrm{id}}\right)^{(k)}(\tau)\right\|_{\mathcal{L}\left(V_{\theta}, V\right)} \leq C d^{k+1 / 2-\theta} \sqrt{\Gamma(2 k+2-2 \theta)} \tau^{-(k+1)+\theta} .
$$

By the representation (3.34) we obtain that

$$
\|U(\tau, \cdot)\|_{V}+\tau\left\|\frac{\partial U}{\partial \tau}(\tau, \cdot)\right\|_{V} \leq C \tau^{\theta} \mathrm{e}^{\lambda \tau}\|f\|_{V_{\theta}} .
$$

\section{LOCALizATion}

Our numerical solution of (3.14)-(3.17) will require truncation of the range $\mathbb{R}$ of log returns to a bounded computational domain $\Omega_{R}=(-R, R)$. Likewise, certain types of contracts (no-touch, touch-and-out) directly lead to the PIDE on the bounded domain. Here, we formulate the PIDE on the bounded domain $\Omega_{R}$ and establish its well-posedness.

In the Black-Scholes case, the localization error can be estimated by local considerations near $\partial \Omega_{R}$ and a maximum principle (see, e.g., [22]). For the PIDE (4.1)-(4.3), local arguments do not apply and we resort to the weighted norm estimates for the PIDE to control the domain truncation error.

\subsection{PIDE on the bounded domain}

Instead of solving (3.30) and (3.31) in $J \times \mathbb{R}$, where we denote by $J$ the time interval $J=(0, T)$, we solve the following problem for the excess to "transformed" payoff in $J \times \Omega_{R}$ :

$$
\begin{aligned}
\frac{\partial U_{R}}{\partial \tau}+\mathcal{A}_{R}\left[U_{R}\right] & =-\left.\mathcal{A}[h]\right|_{\Omega_{R}} \quad \text { in } \quad J \times \Omega_{R} \\
\left.U_{R}(\tau, \cdot)\right|_{\partial \Omega_{R}}=0 & \text { on } \quad \partial \Omega_{R} \quad \forall 0<\tau \leq T \\
\left.U_{R}\right|_{\tau=0}=0 & \text { in } \quad \Omega_{R},
\end{aligned}
$$

with $\mathcal{A}_{R}$ denoting the restriction of $\mathcal{A}$ to $\Omega_{R}$. It is defined as follows: for any function $u$ with support in $\Omega_{R}$, denote by $\tilde{u}$ its extension by zero to all of $\mathbb{R}$. Based on $a(\cdot, \cdot)$ in $(3.23)$, we define

$$
a_{R}(u, v):=a(\tilde{u}, \tilde{v}), \quad u, v \in H_{0}^{1}\left(\Omega_{R}\right) .
$$

Then $a_{R}(\cdot, \cdot): H_{0}^{1}\left(\Omega_{R}\right) \times H_{0}^{1}\left(\Omega_{R}\right) \mapsto \mathbb{R}$, induces $\mathcal{A}_{R}: H_{0}^{1}\left(\Omega_{R}\right) \rightarrow H^{-1}\left(\Omega_{R}\right)=\left(H_{0}^{1}\left(\Omega_{R}\right)\right)^{*}$ via

$$
a_{R}(\varphi, \psi):=\left\langle\mathcal{A}_{R}[\varphi], \psi\right\rangle_{H^{-1}\left(\Omega_{R}\right) \times H_{0}^{1}\left(\Omega_{R}\right)}=\langle\mathcal{A}[\tilde{\varphi}], \tilde{\psi}\rangle_{\left(H^{1}(\mathbb{R})\right)^{*} \times H^{1}(\mathbb{R})} \quad \forall \varphi, \psi \in H_{0}^{1}\left(\Omega_{R}\right) .
$$


Note that, unlike in the Black-Scholes case, the non-local operator $\mathcal{A}$ requires the pay-off $h$ also outside of $\Omega_{R}$. To cast (4.1)-(4.3) into the parabolic setting, we select $V:=H_{0}^{1}\left(\Omega_{R}\right)$ and we identify $L^{2}\left(\Omega_{R}\right)$ with its dual so that $V \stackrel{d}{\hookrightarrow} L^{2}\left(\Omega_{R}\right) \stackrel{d}{\hookrightarrow} V^{*}$ with dense embeddings and with $V^{*}=H^{-1}\left(\Omega_{R}\right)$.

The variational formulation of (4.1)-(4.3) reads: given $f:=-\left.\mathcal{A}[h]\right|_{\Omega_{R}} \in H^{-1}\left(\Omega_{R}\right)$, find $U_{R} \in L^{2}(J, V) \cap$ $H^{1}\left(J, V^{*}\right)$ such that $U_{R}(0)=0$ and such that for every $v \in V$ and every $\varphi \in C_{0}^{\infty}(J)$

$$
-\int_{J}\left(U_{R}(\tau, \cdot), v\right)_{L^{2}\left(\Omega_{R}\right)} \varphi^{\prime}(\tau) \mathrm{d} \tau+\int_{J} a_{R}\left(U_{R}(\tau, \cdot), v\right) \varphi(\tau) \mathrm{d} \tau=\langle f, v\rangle_{V^{*} \times V},
$$

where by $\langle\cdot, \cdot\rangle_{V^{*} \times V}$ we denote the extension of $(\cdot, \cdot)_{L^{2}\left(\Omega_{R}\right)}$ as duality pairing in $V^{*} \times V$. By Theorem 3.4 with $\eta=0$, there exist $C>0$ and $\alpha>0, \beta \geq 0$ such that

$$
\begin{aligned}
& \forall \varphi, \psi \in V:\left|a_{R}(\varphi, \psi)\right| \leq C\|\varphi\|_{V}\|\psi\|_{V} \\
& \forall \varphi \in V: a_{R}(\varphi, \varphi)+\beta\|\varphi\|^{2} \geq \alpha\|\varphi\|_{V}^{2} .
\end{aligned}
$$

Without loss of generality we assume from now on that the bilinear form $a_{R}$ is positive on $V \times V$, since by the substitution

$$
V_{R}=\mathrm{e}^{-\beta \tau} U_{R}
$$

$V_{R}$ solves

$$
\frac{\mathrm{d}}{\mathrm{d} \tau} V_{R}+\left(\mathcal{A}_{R}+\beta \cdot \mathrm{id}\right) V_{R}=\mathrm{e}^{-\beta \tau} f \quad \text { in } J
$$

and the operator $\mathcal{A}_{R}+\beta \cdot$ id is, by (4.8), coercive. Hence, by Theorem 2.3 there exists a unique solution of $(4.1)-(4.3)$.

\subsection{Localization error estimates}

The restriction of the excess to "transformed" payoff $U$ from $\mathbb{R}$ to $\Omega_{R}$ introduces a localization error $e_{R}:=$ $\tilde{U}_{R}-U$ which we now estimate. Since we work in "transformed" quantities, $r=0$ throughout.

Theorem 4.1. Let $\Omega_{R / 2}:=\{|x| \leq R / 2\}$. Then there exist positive constants $C=C(T), \alpha>0$ independent of $R$ such that the localization error $e_{R}=U_{R}-U$ satisfies for all $R>0$

$$
\left\|e_{R}(\tau, \cdot)\right\|_{L^{2}\left(\Omega_{R / 2}\right)}^{2}+\int_{0}^{\tau}\left\|e_{R}(s, \cdot)\right\|_{H^{1}\left(\Omega_{R / 2}\right)}^{2} \mathrm{~d} s \leq C \mathrm{e}^{-\alpha R} .
$$

Proof. Take the weighting exponent $\eta>0$ as in (3.26) and (3.27). Inserting $v=U(\tau)$ in $(3.32)$ and (3.33) and integrating from 0 to $\tau$ implies the following a priori estimate

$$
\|U(\tau)\|_{L_{\eta}^{2}(\mathbb{R})}^{2}+\int_{0}^{\tau}\|U(s)\|_{H_{\eta}^{1}(\mathbb{R})}^{2} \mathrm{~d} s \leq C\|f\|_{\left(H_{\eta}^{1}(\mathbb{R})\right)^{*}}^{2} \quad \forall \tau \in(0, T)
$$

for some constant $C=C(T)>0$ independent $C=C(T)>0$ of $R$. Likewise,

$$
\| \tilde{U}_{R}\left(\tau\left\|_{L_{\eta}^{2}(\mathbb{R})}^{2}+\int_{0}^{\tau}\right\| \tilde{U}_{R}(s)\left\|_{H_{\eta}^{1}(\mathbb{R})}^{2} \mathrm{~d} s \leq C\right\| f \|_{\left(H_{\eta}^{1}(\mathbb{R})\right)^{*}}^{2} \quad \forall \tau \in(0, T)\right.
$$

with same constant $C$ as in (4.11). In particular, $C$ is independent of $R$. Note also that the error $e_{R}=\tilde{U}_{R}-U$ satisfies on $\mathbb{R}$

$$
\left(\frac{\mathrm{d}}{\mathrm{d} \tau} e_{R}(\tau), \tilde{v}\right)_{L^{2}(\mathbb{R})}+a\left(e_{R}(\tau), \tilde{v}\right)=0 \quad \forall v \in H_{0}^{1}\left(\Omega_{R}\right)
$$


Denote by $\phi$ a cut-off function with the properties: $\phi \in C_{0}^{\infty}\left(\Omega_{R}\right), \phi \equiv 1$ on $\Omega_{R / 2}$ and $\left\|\phi^{\prime}\right\|_{L^{\infty}\left(\Omega_{R}\right)} \leq C$ for some constant $C>0$ independent of $R$.

Inserting $v=\phi^{2}(x) e_{R}(\tau, x)$ in $(4.13)$ we obtain

$$
\frac{1}{2} \frac{\mathrm{d}}{\mathrm{d} \tau}\left\|\phi e_{R}(\tau)\right\|_{L^{2}\left(\Omega_{R}\right)}^{2}+a_{R}\left(\phi e_{R}(\tau), \phi e_{R}(\tau)\right)=\rho_{R}(\tau)
$$

where the residual $\rho_{R}(\tau)$ is given by $\rho_{R}(\tau):=a_{R}\left(\phi e_{R}(\tau), \phi e_{R}(\tau)\right)-a\left(e_{R}(\tau), \phi^{2} e_{R}(\tau)\right)$. We observe that

$$
\rho_{R}(\tau)=\frac{\sigma^{2}}{2} \int_{\mathbb{R}}\left|\phi^{\prime}(x)\right|^{2}\left|e_{R}(\tau, x)\right|^{2} \mathrm{~d} x+\left(\frac{\sigma^{2}}{2}+c_{\exp }^{\prime}\right) \int_{\mathbb{R}} \phi^{\prime}(x) \phi(x)\left|e_{R}(\tau, x)\right|^{2} \mathrm{~d} x+\bar{\rho}_{R}(\tau)
$$

where we denote by $\bar{\rho}_{R}(\tau)$ the residual

$$
\bar{\rho}_{R}(\tau)=\bar{a}\left(\phi e_{R}(\tau), \phi e_{R}(\tau)\right)-\bar{a}\left(e_{R}(\tau), \phi^{2} e_{R}(\tau)\right)
$$

from the bilinear form $\bar{a}(\varphi, \phi):=\int_{\mathbb{R}} \bar{A}[\varphi](x) \phi(x) \mathrm{d} x$ with $\bar{A}$ defined by

$$
\varphi \mapsto \bar{A}[\varphi](x):=-\int_{\mathbb{R}}\left\{\varphi(x+y)-\varphi(x)-y \varphi^{\prime}(x)\right\} k(y) \mathrm{d} y
$$

and by $c_{\exp }^{\prime}$ the constant

$$
c_{\exp }^{\prime}=\int_{\mathbb{R}}\left\{e^{y}-1-y\right\} k(y) \mathrm{d} y .
$$

The first two integral terms in the expression (4.15) of the residual $\rho_{R}(\tau)$ are supported in $\Omega_{R} \backslash \Omega_{R / 2}$ and can be estimated by

$$
\begin{aligned}
\left.\left|\frac{\sigma^{2}}{2} \int_{\mathbb{R}}\right| \phi^{\prime}(x)\right|^{2}\left|e_{R}(\tau, x)\right|^{2} \mathrm{~d} x+\left(\frac{\sigma^{2}}{2}+c_{\exp }^{\prime}\right) & \int_{\mathbb{R}} \phi^{\prime}(x) \phi(x)\left|e_{R}(\tau, x)\right|^{2} \mathrm{~d} x \mid \\
& \leq C \int_{\Omega_{R} \backslash \Omega_{R / 2}}\left|e_{R}(\tau, x)\right|^{2} \mathrm{e}^{\eta(x)} \mathrm{e}^{-\eta(x)} \mathrm{d} x \leq C \mathrm{e}^{-\alpha R}\left\|e_{R}(\tau)\right\|_{L_{\eta}^{2}(\mathbb{R})}^{2},
\end{aligned}
$$

for some positive constants $C, \alpha$ independent of $R$.

It remains to estimate the residual $\bar{\rho}_{R}(\tau)$. To this end, let us denote by $k^{(-1)}$ the first anti-derivative of the Lévy kernel $k$ vanishing as $|x| \rightarrow \infty$

$$
k^{(-1)}(x)=\left\{\begin{array}{c}
-\int_{x}^{\infty} k(y) \mathrm{d} y \text { if } x>0 \\
\int_{-\infty}^{x} k(y) \mathrm{d} y \text { if } x<0 .
\end{array}\right.
$$

Observe that for $k$ satisfying (A1)-(A3) with $Y<2, G>0$ and $M>1$ the first antiderivative kernel $k^{(-1)}$ has the same rate of exponential decay as $k$ as $\pm x \rightarrow \infty$ and $y k^{(-1)}(y)$ is in $L^{1}(\mathbb{R})$.

Integration by parts implies for $\bar{A}$ as in (4.17) the representation

$$
\bar{A}[\varphi](x)=\int_{\mathbb{R}}\left\{\varphi^{\prime}(x+y)-\varphi^{\prime}(x)\right\} k^{(-1)}(y) \mathrm{d} y \quad \forall \varphi \in H^{1}(\mathbb{R}) .
$$


With these notations we get from (4.16),

$$
\begin{aligned}
\bar{\rho}_{R}(\tau)= & \iint_{\mathbb{R}^{2}}\left(\frac{\partial e_{R}}{\partial x}(\tau, x+y) \phi(x+y)+e_{R}(\tau, x+y) \phi^{\prime}(x+y)\right. \\
& \left.-\frac{\partial e_{R}}{\partial x}(\tau, x) \phi(x)-e_{R}(\tau, x) \phi^{\prime}(x)\right) k^{(-1)}(y) e_{R}(\tau, x) \phi(x) \mathrm{d} y \mathrm{~d} x \\
& -\iint_{\mathbb{R}^{2}}\left(\frac{\partial e_{R}}{\partial x}(\tau, x+y)-\frac{\partial e_{R}}{\partial x}(\tau, x)\right) k^{(-1)}(y) e_{R}(\tau, x) \phi^{2}(x) \mathrm{d} y \mathrm{~d} x
\end{aligned}
$$

or, equivalently,

$$
\begin{aligned}
\bar{\rho}_{R}(\tau)= & \iint_{\mathbb{R}^{2}} \frac{\partial e_{R}}{\partial x}(\tau, x+y)(\phi(x+y)-\phi(x)) k^{(-1)}(y) e_{R}(\tau, x) \phi(x) \mathrm{d} y \mathrm{~d} x \\
& +\iint_{\mathbb{R}^{2}}\left(e_{R}(\tau, x+y) \phi^{\prime}(x+y)-e_{R}(\tau, x) \phi^{\prime}(x)\right) k^{(-1)}(y) e_{R}(\tau, x) \phi(x) \mathrm{d} y \mathrm{~d} x \\
= & I_{1}+I_{2} .
\end{aligned}
$$

We observe that the integrand in the first term of $\rho_{R}(\tau)$ in (4.19) is supported by $|x+y| \geq R / 2$ or $|x| \geq R / 2$ (otherwise $\phi(x+y)-\phi(x)=1-1=0$ ), i.e.,

$$
I_{1}=\iint_{\{|x+y| \geq R / 2\} \cup\{|x| \geq R / 2\}} \frac{\partial e_{R}}{\partial x}(\tau, x+y)(\phi(x+y)-\phi(x)) k^{(-1)}(y) e_{R}(\tau, x) \phi(x) \mathrm{d} y \mathrm{~d} x .
$$

It implies that

$$
\begin{aligned}
\left|I_{1}\right| \leq & C \iint_{|x+y| \geq R / 2}\left|\frac{\partial e_{R}}{\partial x}(\tau, x+y)\right| \mathrm{e}^{\eta(x+y)} \mathrm{e}^{-\eta(x+y)}|y|\left|k^{(-1)}(y)\right|\left|e_{R}(\tau, x)\right| \phi(x) \mathrm{d} y \mathrm{~d} x \\
& +\iint_{|x| \geq R / 2}\left|\frac{\partial e_{R}}{\partial x}(\tau, x+y)\right||y|\left|k^{(-1)}(y) \| e_{R}(\tau, x)\right| \mathrm{e}^{\eta(x)} \mathrm{e}^{-\eta(x)} \mathrm{d} y \mathrm{~d} x \\
\leq & C \mathrm{e}^{-\alpha R}\left(\left\|\frac{\partial e_{R}}{\partial x}(\tau)\right\|_{L_{\eta}^{2}(\mathbb{R})}\left\|e_{R}(\tau)\right\|_{L^{2}(\mathbb{R})}+\left\|\frac{\partial e_{R}}{\partial x}(\tau)\right\|_{L^{2}(\mathbb{R})}\left\|e_{R}(\tau)\right\|_{L_{\eta}^{2}(\mathbb{R})}\right)
\end{aligned}
$$

for some positive constants $C, \alpha$ independent of $R$. Analogous reasoning applies to the second integral term in (4.19) after we split it into

$$
\begin{aligned}
I_{2}= & \iint_{\mathbb{R}^{2}}\left(e_{R}(\tau, x+y)-e_{R}(\tau, x)\right) \phi^{\prime}(x) k^{(-1)}(y) e_{R}(\tau, x) \phi(x) \mathrm{d} y \mathrm{~d} x \\
& +\iint_{\mathbb{R}^{2}} e_{R}(\tau, x+y)\left(\phi^{\prime}(x+y)-\phi^{\prime}(x)\right) k^{(-1)}(y) e_{R}(\tau, x) \phi(x) \mathrm{d} y \mathrm{~d} x=I_{21}+I_{22} .
\end{aligned}
$$

The integrand function in the first integral $I_{21}$ in (4.21) is supported on $\{|x| \geq R / 2\}$, so that $I_{21}$ can be estimated as follows:

$$
\begin{aligned}
\left|I_{21}\right| & =\left|\iint_{|x| \geq R / 2}\left(e_{R}(\tau, x+y)-e_{R}(\tau, x)\right) \phi^{\prime}(x) k^{(-1)}(y) e_{R}(\tau, x) \mathrm{e}^{\eta(x)} \mathrm{e}^{-\eta(x)} \phi(x) \mathrm{d} y \mathrm{~d} x\right| \\
& \leq C \mathrm{e}^{-\alpha R}\left\|e_{R}(\tau)\right\|_{H^{1}(\mathbb{R})}\left\|e_{R}(\tau)\right\|_{H_{\eta}^{1}(\mathbb{R})} .
\end{aligned}
$$


The term $I_{22}$ in (4.21) can be treated similarly to $I_{1}$ and satisfies

$$
\left|I_{22}\right| \leq C \mathrm{e}^{-\alpha R}\left\|e_{R}(\tau)\right\|_{L_{\eta}^{2}(\mathbb{R})}\left\|e_{R}(\tau)\right\|_{L^{2}(\mathbb{R})} .
$$

Integrating (4.14) from 0 to $\tau$ and using the estimates for $\rho_{R}(\tau)$ from (4.18), (4.20), (4.22) and (4.23) together with the a priori estimates (4.11)-(4.12) yields (4.10).

\subsection{Pure jump case: $\boldsymbol{\sigma}=\mathbf{0}$}

In Section 4.1 we introduced the PIDE on $\Omega_{R}$ for $\sigma>0$ and discussed its well-posedness in the space $V=H_{0}^{1}\left(\Omega_{R}\right)$. To cast the PIDE in the case $\sigma=0$ of pure jump processes into the abstract form (2.12), we require for $0 \leq s \leq 1$ the spaces [1]

$$
\tilde{H}^{s}\left(\Omega_{R}\right)=\left\{\left.u\right|_{\Omega_{R}}\left|u \in H^{s}(\mathbb{R}), u\right|_{\mathbb{R} \backslash \Omega_{R}}=0\right\} .
$$

For $s=0$ we have $\tilde{H}^{s}\left(\Omega_{R}\right)=L^{2}\left(\Omega_{R}\right)$, for $s=1$ we have $\tilde{H}^{s}\left(\Omega_{R}\right)=H_{0}^{1}\left(\Omega_{R}\right)$. In the case $0<s<1$ we define the norm $\|v\|_{\tilde{H}^{s}\left(\Omega_{R}\right)}$ by

$$
\|v\|_{\tilde{H}^{s}\left(\Omega_{R}\right)}^{2}=\|v\|_{L^{2}\left(\Omega_{R}\right)}^{2}+|v|_{\tilde{H}^{s}\left(\Omega_{R}\right)}^{2}, \quad|v|_{\tilde{H}^{s}\left(\Omega_{R}\right)}^{2}=\int_{\mathbb{R}} \int_{\mathbb{R}} \frac{|\tilde{v}(x)-\tilde{v}(y)|^{2}}{|x-y|^{1+2 s}} \mathrm{~d} x \mathrm{~d} y
$$

where we recall that $\tilde{v}(x)$ denotes the extension of $v(x)$ by zero for $x \in \mathbb{R}$. Note that one can use for $s \in(0,1)$, $s \neq \frac{1}{2}$ in (4.25) integrals over $\Omega_{R}$ instead of $\mathbb{R}$. For $s=\frac{1}{2}$, however, which frequently occurs in practice (e.g. $[5,18,40]$ ), using the integral over $\Omega_{R}$ in (4.25) would give the norm for the space $H^{1 / 2}\left(\Omega_{R}\right)$ which is different from $\tilde{H}^{1 / 2}\left(\Omega_{R}\right)$ : in fact, $\tilde{H}^{1 / 2}\left(\Omega_{R}\right)=H_{00}^{1 / 2}\left(\Omega_{R}\right)$ and (see [26]),

$$
\|v\|_{\tilde{H}^{1 / 2}\left(\Omega_{R}\right)}^{2}=\|v\|_{L^{2}\left(\Omega_{R}\right)}^{2}+\int_{\Omega_{R}} \int_{\Omega_{R}} \frac{|v(x)-v(y)|^{2}}{|x-y|^{2}} \mathrm{~d} x \mathrm{~d} y+\int_{\Omega_{R}} \frac{|v(x)|^{2}}{R^{2}-x^{2}} \mathrm{~d} x .
$$

We show now that for $\sigma=0$ the PIDE (3.14) fits, for $0<Y<2$, into the abstract parabolic framework (2.8)-(2.12) based on

$$
V=\tilde{H}^{Y / 2}\left(\Omega_{R}\right) \hookrightarrow L^{2}\left(\Omega_{R}\right) \hookrightarrow V^{*}=H^{-Y / 2}\left(\Omega_{R}\right)
$$

We begin by showing the Gårding inequality.

Proposition 4.2. Assume that $X_{t}$ is a pure jump Lévy process with Lévy density $k$ satisfying (A1)-(A4) for some $0<Y<2$. Then, there exist two positive constants $c_{1}=c_{1}(R)>0$ and $c_{2}=c_{2}(R)>0$ such that

$$
\forall u \in \tilde{H}^{Y / 2}\left(\Omega_{R}\right): a_{R}(u, u) \geq c_{1}\|u\|_{\tilde{H}^{Y / 2}\left(\Omega_{R}\right)}^{2}-c_{2}\|u\|_{L^{2}\left(\Omega_{R}\right)}^{2} .
$$

Proof. By a localization argument and (A2), (A3), we may assume that $R=1 / 2$ and, by density, that $u \in$ $C_{0}^{\infty}\left(\Omega_{R}\right)$. Then $\left(u^{\prime}, u\right)=0$. As before, we denote by $\tilde{u}$ the extension of $u$ by zero to $\mathbb{R}$. By $a_{R}^{s}(\varphi, \psi)=$ $\left(a_{R}(\varphi, \psi)+a_{R}(\psi, \varphi)\right) / 2$ we denote the bilinear form for the symmetric part $\hat{A}^{s}:=\frac{1}{2}\left(\hat{A}+\hat{A}^{*}\right)=\frac{1}{2}\left(A+A^{*}\right)=: A^{s}$ of $\hat{A}$. Noting that $\hat{A}[1]=0, A[1]=0$, we find

$$
a_{R}(u, u)=a_{R}^{s}(u, u)=\left\langle A^{s} \tilde{u}, \tilde{u}\right\rangle=\int_{\mathbb{R}} \int_{\mathbb{R}} \frac{1}{2}[k(x-y)+k(y-x)]|\tilde{u}(x)-\tilde{u}(y)|^{2} \mathrm{~d} y \mathrm{~d} x .
$$


Due to $R=1 / 2, x, y \in \operatorname{supp}(\tilde{u}) \subseteq \bar{\Omega}_{R}$ implies $|x-y|<1$ and we obtain from (A4), (4.25)

$$
a_{R}(\tilde{u}, \tilde{u}) \geq C_{-} \int_{\mathbb{R}} \int_{\mathbb{R}} \frac{|\tilde{u}(x)-\tilde{u}(y)|^{2}}{|x-y|^{1+Y}} \mathrm{~d} y \mathrm{~d} x=C_{-}\left(\|u\|_{\tilde{H}^{Y / 2}\left(\Omega_{R}\right)}^{2}-\|u\|_{L^{2}\left(\Omega_{R}\right)}^{2}\right)
$$

which implies (4.26).

Proposition 4.2 gives the Gårding inequality $(2.11)$ for $a_{R}(\cdot, \cdot)$ in the pure jump case $\sigma=0$ for all values of $Y \in(0,2)$.

The continuity $a_{R}(\cdot, \cdot)$ on $\tilde{H}^{Y / 2}\left(\Omega_{R}\right) \times \tilde{H}^{Y / 2}\left(\Omega_{R}\right)$ in the pure jump case $\sigma=0$ is obtained as follows: for $u, v \in C_{0}^{\infty}\left(\Omega_{R}\right)$, we have in "transformed" variables that $r=0$ and

$$
a_{R}(u, v)=\langle A \tilde{u}, \tilde{v}\rangle+c_{\exp }\left\langle\tilde{u}^{\prime}, \tilde{v}\right\rangle .
$$

By using (A1) and the Fourier transform, the operator $A: H^{Y / 2}(\mathbb{R}) \rightarrow H^{-Y / 2}(\mathbb{R})$ boundedly for $Y \geq 1$. For the drift term, we estimate for $Y \geq 1$

$$
\left|\left\langle\tilde{u}^{\prime}, \tilde{v}\right\rangle\right|=c|\langle i \xi \hat{\tilde{u}}, \hat{\tilde{v}}\rangle| \leq c\|\tilde{u}\|_{H^{1 / 2}(\mathbb{R})}\|\tilde{v}\|_{H^{1 / 2}(\mathbb{R})} \leq C\|u\|_{\tilde{H}^{Y / 2}\left(\Omega_{R}\right)}\|v\|_{\tilde{H}^{Y / 2}\left(\Omega_{R}\right)}
$$

which implies the continuity of $a_{R}(\cdot, \cdot)$ for $Y \geq 1$. Therefore, (2.10) holds for $\sigma=0$ with $V=\tilde{H}^{Y / 2}\left(\Omega_{R}\right)$ for $1 \leq Y \leq 2$.

\subsection{Removal of drift}

In order to prove $(2.10)$ for $a_{R}(\cdot, \cdot)$ in the case $\sigma=0$ for $0<Y<1$, it is important that we can transform (4.1)-(4.3) to remove the drift term so that the transformed equation will still satisfy (2.11) in $V=\tilde{H}^{Y / 2}\left(\Omega_{R}\right)$ and also the continuity estimate (2.10) for all $0<Y<2$ for $\sigma=0$. As our numerical scheme below exploits the parabolic nature of (3.14), numerical instabilities might result from a dominant first order term, even if $\sigma \neq 0$, so that removal of drift is also of interest then. To this end, recall

$$
\mathcal{A}_{R}[u]=-\frac{\sigma^{2}}{2} u_{x x}+\left(\frac{\sigma^{2}}{2}-r\right) u_{x}+r u+\hat{A}_{R}[u],
$$

with $\hat{A}_{R}$ being the restriction to $\Omega_{R}$ of the integro-differential operator $\hat{A}$ in (3.29). By the reduction (2.21) to "transformed" variables, $r=0$ and we also assume $Y<1$. Then the operator $\hat{A}_{R}$ in (4.27) can be written as the sum of a first order term and an integro-differential operator $\mathcal{C}$ which is continuous and coercive on $\tilde{H}^{Y / 2}\left(\Omega_{R}\right)$ : for every $\varphi, \psi \in C_{0}^{\infty}\left(\Omega_{R}\right)$ holds

$$
\left\langle\psi, \hat{A}_{R}[\varphi]\right\rangle=c_{1}\left\langle\psi, \varphi^{\prime}\right\rangle-\int_{\mathbb{R}} \int_{\mathbb{R}} \tilde{\psi}(x) k(y-x)(\tilde{\varphi}(y)-\tilde{\varphi}(x)) \mathrm{d} y \mathrm{~d} x=: c_{1}\left\langle\psi, \varphi^{\prime}\right\rangle+\langle\psi, \mathcal{C} \varphi\rangle
$$

with

$$
c_{1}:=\int_{y \in \mathbb{R}}\left(\mathrm{e}^{y}-1\right) k(y) \mathrm{d} y .
$$

Note that $\left\langle\psi, \hat{A}_{R}[\exp ]\right\rangle=0$ for all $\psi$ and, as before, by (A1) it holds

$$
|\langle\psi, \mathcal{C} \varphi\rangle| \leq C\|\psi\|_{\tilde{H}^{Y / 2}\left(\Omega_{R}\right)}\|\varphi\|_{\tilde{H}^{Y / 2}\left(\Omega_{R}\right)}
$$

and, arguing as before, by (A4) there are $C_{1}, C_{2}>0$ such that

$$
\forall \varphi \in \tilde{H}^{Y / 2}\left(\Omega_{R}\right): \quad\langle\varphi, \mathcal{C} \varphi\rangle \geq C_{1}\|\varphi\|_{\tilde{H}^{Y / 2}\left(\Omega_{R}\right)}^{2}-C_{2}\|\varphi\|_{L^{2}\left(\Omega_{R}\right)}^{2} .
$$


We remove the term $c_{1} \varphi^{\prime}$ in $\hat{A}_{R}$ (which obstructs $\tilde{H}^{Y / 2}\left(\Omega_{R}\right)$-continuity of $\left\langle\psi, \hat{A}_{R}[\varphi]\right\rangle$ ) by

$$
U_{R}(\tau, x)=V_{R}\left(\tau, x-\left(\sigma^{2} / 2+c_{1}\right) \tau\right)
$$

This yields in (4.1)-(4.3) the equation

$$
\begin{aligned}
\frac{\partial V_{R}}{\partial \tau}-\frac{\sigma^{2}}{2} \frac{\partial^{2} V_{R}}{\partial x^{2}}+r V_{R}+\mathcal{C}\left[V_{R}\right] & =\left(-\left.\mathcal{A}[h]\right|_{\Omega_{R}}\right)\left(x+\left(\sigma^{2} / 2-r+c_{1}\right) \tau\right), \\
V_{R}(0, x) & =0 \quad \text { in } \Omega_{R} .
\end{aligned}
$$

Hence, for $0<Y<2, \sigma \geq 0, r \geq 0$ and under (A4) if $\sigma=0$ the bilinear form $a_{R}(\cdot, \cdot): V \times V \rightarrow \mathbb{R}$ satisfies (2.10), (2.11) (cf. Prop. 4.2) in the Gelfand triple $V \stackrel{d}{\hookrightarrow} L^{2}\left(\Omega_{R}\right) \stackrel{d}{\hookrightarrow} V^{*}$ with

$$
V:=\tilde{H}^{\rho / 2}\left(\Omega_{R}\right) \quad \text { where } \quad \rho= \begin{cases}2 & \text { if } \sigma>0 \\ Y & \text { if } \sigma=0\end{cases}
$$

\section{Numerical SOLUtion}

We obtain on the bounded domain $\Omega_{R}=(-R, R)$ the following parabolic problem for $\tau \in[0, T]$ : Find $U \in L^{2}([0, T], V) \cap H^{1}\left([0, T], V^{*}\right)$ such that

$$
\begin{aligned}
\left\langle\frac{\mathrm{d}}{\mathrm{d} \tau} U, v\right\rangle+a_{R}(U, v) & =\langle f, v\rangle_{V^{*} \times V} \quad \text { for all } v \in V \\
U(0) & =0
\end{aligned}
$$

with $V$ as in (4.33) and $a_{R}(\phi, \psi)=a(\tilde{\phi}, \tilde{\psi})$ given by $(3.23)$ where $\tilde{\phi}, \tilde{\psi}$ denote the extensions by zero. Because of the transformation (2.21) we assume that $r=0$. Here the function $U=u-h$ is the excess to payoff and the functional $f \in V^{*}$ is given by $\langle f, \phi\rangle:=-a(h, \tilde{\phi})$.

In the case $\sigma=0$ and $Y<1$ we obtain after the additional transformation in Section 4.4 a problem of form (5.1), (5.2) with $a_{R}(\phi, \psi):=\langle\mathcal{C}[\tilde{\phi}], \tilde{\psi}\rangle$ and $f$ given by $\langle f(\tau), \phi\rangle:=-a\left(h\left(\cdot+c_{1} \tau\right), \tilde{\phi}\right)$.

In all cases the bilinear form $a_{R}$ satisfies (2.10), (2.11), therefore the problem (5.1), (5.2) has a unique solution by Theorem 2.3 .

\subsection{Non-translation-invariant operators}

So far we discussed the case where the $\log$-price $X_{t}=\log S_{t}$ is a Lévy process under the risk-neutral measure. Our numerical method, however, applies also to the more general case where the log-price process $X_{t}$ is a Markov process where the increments $X_{t}-X_{s}$ are no longer independent of $X_{s}$ for $t>s$. In this case we can have a volatility $\sigma(x)$ depending on $x$, and the singular jump measure can also depend on $x$. Instead of the translation-invariant operator $\mathcal{A}$ from (3.21) with $A[\phi]$ defined by (3.15) we now consider a more general operator $\mathcal{A}$ which may depend on $x$ : Let

$$
\mathcal{A}[\varphi](x):=-\frac{\sigma(x)^{2}}{2} \frac{\mathrm{d}^{2} \varphi}{\mathrm{d} x^{2}}(x)+\left(\frac{\sigma(x)^{2}}{2}-r\right) \frac{\mathrm{d} \varphi}{\mathrm{d} x}(x)+r \varphi+\hat{A}[\varphi](x)
$$

with the integral operator $\hat{A}[\phi]$ given by

$$
\begin{aligned}
A[\phi](x) & :=-\int_{\mathbb{R}}\left\{\phi(y)-\phi(x)-(y-x) \phi^{\prime}(x) \chi_{[-1,1]}(y-x)\right\} k(x, y-x) \mathrm{d} y, \\
\hat{A}[\phi] & :=A[\phi](x)+c_{\exp }(x) \phi^{\prime}(x)
\end{aligned}
$$


and $c_{\exp }(x)$ chosen such that $\hat{A}[\exp (\cdot)]=0$. We assume that $\sigma(x)$ is bounded for all $x \in \Omega_{R}$

$$
0<\sigma_{0} \leq \sigma(x) \leq \sigma_{1}
$$

and that $k(x, z)$ satisfies assumptions (A1), (A2) uniformly for $x \in \Omega_{R}$. Instead of (A3) we require the CalderónZygmund estimates: for all $\alpha, \beta \in \mathbb{N}_{0}$ there holds for $z \neq 0$

$$
\left|\partial_{x}^{\alpha} \partial_{z}^{\beta} k(x, z)\right| \leq C(\alpha, \beta)|z|^{-(1+Y+\alpha+\beta)} .
$$

In the case $\sigma=0$ we require (A4) uniformly for $x \in \Omega_{R}$.

We define $U(\tau, x):=u(\tau, x)-\mathrm{e}^{-r \tau} h(x+r \tau)$ as the excess to the transformed payoff (cf. Sect. 4.1) and obtain the parabolic evolution problem $(5.1),(5.2)$ with $a_{R}(\phi, \psi):=\langle\mathcal{A}[\tilde{\phi}], \tilde{\psi}\rangle$ and $\langle f(\tau), \phi\rangle:=-a(h(\cdot+r \tau), \tilde{\phi})$. Then in the case of $\sigma>0$ or $Y \geq 1$ the bilinear form $a_{R}$ satisfies (2.10), (2.11). Hence the problem (5.1), (5.2) has a unique solution by Theorem 2.3. In the case of $\sigma=0$ and $Y<1$ we need to assume that we can transform the problem such that $a_{R}$ satisfies (2.10).

\subsection{Discretization}

For the space discretization we use the Galerkin method with a finite element subspace $V_{h} \subset V$ of piecewise polynomials. We use a uniform mesh with $n$ subintervals of size $h=2 R / n$ on the interval $\Omega_{R}=(-R, R)$ and denote by $p \in \mathbb{N}$ the polynomial degree. We then define $V_{h}$ as the space of continuous piecewise polynomials of degree $p$ on the mesh which vanish at $x=-R$ and at $x=R$.

The semi-discrete problem reads: given $f \in V^{*}$, find $U_{h} \in H^{1}\left(J, V_{h}\right)$ such that

$$
\begin{aligned}
\left(\frac{\mathrm{d}}{\mathrm{d} \tau} U_{h}, v_{h}\right)+a_{R}\left(U_{h}, v_{h}\right) & =\left\langle f, v_{h}\right\rangle_{V^{*} \times V} \quad \text { for all } v_{h} \in V_{h} \\
U_{h}(0) & =0 .
\end{aligned}
$$

For the time discretization we use the $\theta$-scheme with $M$ steps and step size $k=T / M$. The fully discretized method reads as follows: let $U_{h}^{0}=0$. For $m=0,1, \ldots, M-1$ find $U_{h}^{m+1} \in V_{h}$ such that

$$
\left(\frac{U_{h}^{m+1}-U_{h}^{m}}{k}, v_{h}\right)+a_{R}\left(U_{h}^{m+\theta}, v_{h}\right)=\left\langle f, v_{h}\right\rangle_{V^{*} \times V} \quad \text { for all } v_{h} \in V_{h}
$$

holds. Here $U_{h}^{m+\theta}:=\theta U_{h}^{m+1}+(1-\theta) U_{h}^{m}$. In matrix form, (5.9) reads

$$
\left(k^{-1} \mathbf{M}+\theta \mathbf{A}\right) \underline{U}^{m+1}=k^{-1} \mathbf{M} \underline{U}^{m}-(1-\theta) \mathbf{A} \underline{U}^{m}+\underline{f}, \quad m=0,1, \ldots, M-1 .
$$

where $\underline{U}^{m}$ is the coefficient vector of $U_{h}^{m}$ with respect to a basis of $V_{h}$. The matrices $\mathbf{M}, \mathbf{A}$ denote the mass- and stiffness matrix, respectively, with respect to a basis of $V_{h}$. By Remark 3.3, all eigenvalues of $\mathbf{A}$ have positive real part.

\subsection{Wavelet compression}

Due to the nonlocal operator $\mathcal{A}_{R}$ the matrix $\mathbf{A}$ is fully populated, increasing the complexity of the algorithm.

By using a wavelet basis we will obtain a matrix $\mathbf{A}$ where most elements are very small and can be replaced with zero, yielding a sparse matrix $\tilde{\mathbf{A}}$ with only $O(N \log N)$ nonzero elements where $N=\operatorname{dim} V_{h}$. The wavelet basis will also allow optimal preconditioning. 


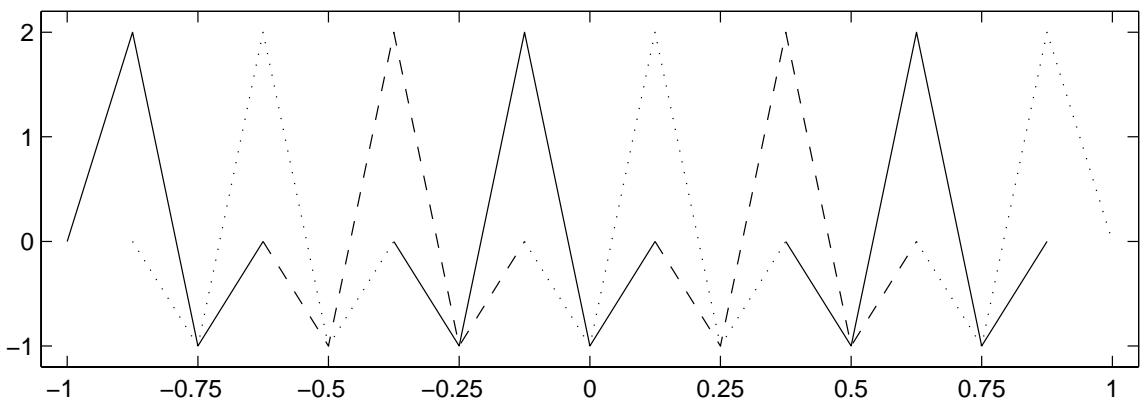

Figure 1. Biorthogonal wavelets $\psi_{j}^{l}$ for $l=3$ (multiplied by $2^{-l / 2}$ ).

\subsubsection{Wavelet basis}

We assume that the number $n$ of subintervals is of the form $n=2^{L} n_{0}, n_{0} \in \mathbb{N}$. For $l=0, \ldots, L$ let $V^{l}$ denote the space of continuous piecewise polynomials of degree $p$ on the uniform mesh with $n_{l}:=2^{l} n_{0}$ intervals which vanish at $x=-R, R$. Then we have

$$
V^{0} \subset V^{1} \subset \cdots \subset V^{L}=V_{h} .
$$

Let $N^{l}:=\operatorname{dim} V^{l}, N^{-1}=0$, and $M^{l}:=N^{l}-N^{l-1}$ for $l=0, \ldots, L$.

It is then possible (see [14]) to construct so-called biorthogonal wavelets $\psi_{j}^{l}$ for $l=0,1, \ldots$, and $j=1, \ldots, M^{l}$ with the following properties:

(P1) The wavelets $\psi_{j}^{l}$ form a hierarchical basis for the spaces $V^{0}, V^{1}, \ldots$ :

$$
V^{l}=\operatorname{span}\left\{\psi_{j}^{k} \mid 1 \leq j \leq M^{k}, 0 \leq k \leq l\right\}
$$

(P2) Wavelets $\psi_{j}^{l}$ with support contained in $(-R, R)$ and $l \geq l_{0}$ have vanishing moments up to order $p$, i.e., $\int \psi_{j}^{l}(x) x^{k} \mathrm{~d} x=0$ for $k=0, \ldots, p$.

(P3) Wavelets $\psi_{j}^{l}(x)$ for $l \geq l_{0}$ are obtained as translates of the functions $2^{\left(l-l_{0}\right) / 2} \psi_{j}^{l_{0}}\left(2^{l-l_{0}} x\right)$. Therefore the support $S_{j}^{l}:=\operatorname{supp}\left(\psi_{j}^{l}\right)$ has diameter of less than $C 2^{-l}$.

(P4) For all $v_{h}=\sum_{l=0}^{L} \sum_{j=1}^{M^{l}} v_{j}^{l} \psi_{j}^{l} \in V_{h}$ there holds the norm equivalence for $s \in[0,1]$

$$
\left\|v_{h}\right\|_{s}^{2}:=\sum_{l=0}^{L} \sum_{j=1}^{M^{l}} 2^{2 l s}\left|v_{j}^{l}\right|^{2} \sim\left\|v_{h}\right\|_{\tilde{H}^{s}\left(\Omega_{R}\right)}^{2} .
$$

Example for $p=1$ : Let $n_{0}=2$. Then $N^{l}=2^{l+1}-1, M^{l}=2^{l}$. A piecewise linear function in $V^{l}$ can be specified by giving the values at the nodes $x_{j}^{l}:=-R+j h, j=1, \ldots, n_{l}-1$.

Define $\psi_{1}^{0}$ by $\psi_{1}^{0}\left(x_{1}\right)=1$. Let now $l \geq l_{0}:=1$. Define $\psi_{1}^{l}$ by the values $2 \cdot 2^{l / 2},-2^{l / 2}$ at $x_{1}, x_{2}$ and 0 at all other nodes. Define $\psi_{M^{l}}^{l}$ by the values $-2^{l / 2}, 2 \cdot 2^{l / 2}$ at $x_{n_{l}-2}, x_{n_{l}-1}$ and 0 at all other nodes. Define $\psi_{j}^{l}$ for $1<j<M^{l}$ by the values $-2^{l / 2}, 2 \cdot 2^{l / 2},-2^{l / 2}$ at the nodes $x_{2 j-2}, x_{2 j-1}, x_{2 j}$ and 0 at all other nodes. The functions $\psi_{1}^{3}, \ldots, \psi_{8}^{3}$ for the interval $[-1,1]$ are shown in Figure 1.

\subsubsection{Matrix compression for $Y \geq 0$}

The bilinear form $a_{R}$ on $V_{h} \times V_{h}$ corresponds to a matrix $\mathbf{A}$ with elements $A_{(l, j),\left(l^{\prime}, j^{\prime}\right)}=a_{R}\left(\psi_{j}^{l}, \psi_{j^{\prime}}^{l^{\prime}}\right)$. Note that $|k(z)|$ decays like $|z|^{-Y-1}$ for small $z$ by (3.6), (5.6).

If we used a standard finite element basis the size of the matrix elements would decay like $d^{-Y-1}$ where $d$ denotes the distance of the supports of the two basis functions. 

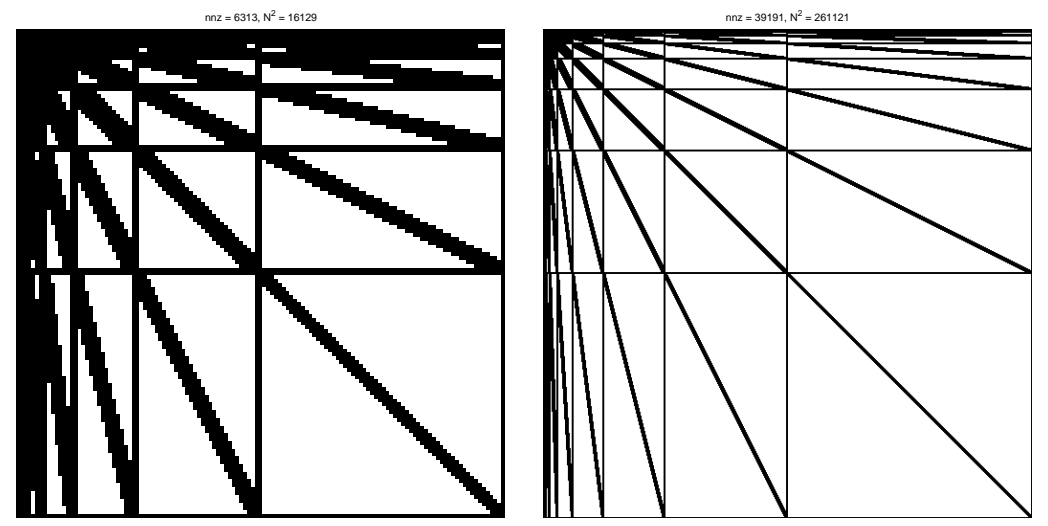

FIGURE 2. Sparsity pattern of the compressed matrix in wavelet basis; CGMY parameters: $C=1.0, Y=1.5, G=0.6, M=2.8 ; N=127$ (left) and $N=511$ (right).

For our wavelet basis $\psi_{j}^{l}$ the vanishing moment property (P4) and (5.6) (with $\alpha+\beta \leq 2 p+2$ ) imply that the entries of the matrix $\mathbf{A}$ actually decay like $d^{-Y-3-2 p}$ where $d=\operatorname{dist}\left(S_{j}^{l}, S_{j^{\prime}}^{l^{\prime}}\right)$. This faster decay allows us to replace most matrix entries with zero without losing accuracy.

We define the compressed matrix $\tilde{\mathbf{A}}$ and the corresponding bilinear form $\tilde{a}_{R}$ by replacing certain small matrix elements in A with zero:

$$
\tilde{A}_{(j, l),\left(j^{\prime}, l^{\prime}\right)}:= \begin{cases}A_{(j, l),\left(j^{\prime}, l^{\prime}\right)} & \text { if } \operatorname{dist}\left(S_{j}^{l}, S_{j^{\prime}}^{l^{\prime}}\right) \leq \delta_{l, l^{\prime}} \text { or } S_{j}^{l} \cap \partial \Omega_{R} \neq \emptyset \\ 0 & \text { otherwise. }\end{cases}
$$

Here the truncation parameters $\delta_{l, l^{\prime}}$ are given by

$$
\delta_{l, l^{\prime}}:=c \max \left\{2^{-L+\hat{\alpha}\left(2 L-l-l^{\prime}\right)}, 2^{-l}, 2^{-l^{\prime}}\right\}
$$

with some parameters $c>0$ and $0<\hat{\alpha} \leq 1$.

Because of (4.9) we can assume that $a_{R}$ is coercive. Therefore it induces a norm which is equivalent to the norm of $V=\tilde{H}^{\rho / 2}\left(\Omega_{R}\right)$ :

$$
\|u\|_{a}:=\left(a_{R}(u, u)\right)^{1 / 2} \sim\|u\|_{V} .
$$

Proposition 5.1. Let $Y \geq 0$. If $c$ in (5.12) is chosen sufficiently large then there exists $0<\delta<1$ independent of $L$ such that for all $L>0$ condition

$$
\left|a_{R}\left(u_{h}, v_{h}\right)-\widetilde{a}_{R}\left(u_{h}, v_{h}\right)\right| \leq \delta\left\|u_{h}\right\|_{a}\left\|v_{h}\right\|_{a} \quad \forall u_{h}, v_{h} \in V_{h}
$$

holds. If additionally

then for all $u_{h}, v_{h} \in V_{h}$

$$
\hat{\alpha} \geq \frac{2 p+2}{2 p+2+Y}
$$

$$
\left|a_{R}\left(u_{h}, v_{h}\right)-\widetilde{a}_{R}\left(u_{h}, v_{h}\right)\right| \leq C h^{p+1-Y / 2}|\log h|^{\nu}\left\|u_{h}\right\|_{p+1}\left\|v_{h}\right\|_{V}
$$

holds with $\nu=1$ if equality holds in (5.14), and $\nu=0$ otherwise.

The matrix compression (5.11) reduces the number of nonzero elements from $N^{2}$ in $\mathbf{A}$ to $N$ times a logarithmic term in $\tilde{\mathbf{A}}$, see Figure 2 and [34]. 
Proposition 5.2. We can choose $\hat{\alpha}$ such that $\nu=0$ in (5.15) and the number of nonzero elements in $\tilde{\mathbf{A}}$ is $O(N \log N)$.

\subsubsection{Matrix compression for $\sigma>0$ and $Y \leq 4-2(p+1)$}

We now consider the case $Y \leq 4-2(p+1)$. Since $Y \leq 0$ we assume $\sigma>0$ and have $\rho=2$. Note that we can write $a_{R}(\phi, \psi)$ using $(4.28)$ as

$$
a_{R}(\phi, \psi)=\int_{\mathbb{R}}\left[\frac{\sigma^{2}}{2} \phi^{\prime}(x) \psi^{\prime}(y)+\left(\frac{\sigma^{2}}{2}+c_{1}\right) \phi^{\prime}(x) \psi(x)\right]+\langle\mathcal{C}[\phi], \psi\rangle=: a_{\mathrm{diff}}(\phi, \psi)+c(\phi, \psi)
$$

where $a_{\text {diff }}(\phi, \psi)$ contains differential operators, and $c(\phi, \psi)$ satisfies (4.29). Therefore we can split the matrix $\mathbf{A}$ corresponding to the bilinear form $a_{R}$ as $\mathbf{A}=\mathbf{A}_{\text {diff }}+\mathbf{C}$. For a standard finite element basis the matrix corresponding to the bilinear form $a_{\text {diff }}$ has $O(N)$ nonzero elements. We can transform a coefficient vector from the standard finite element basis to the wavelet basis and vice versa in $O(N)$ operations. Therefore we can implement the operation $\underline{v} \mapsto \mathbf{A}_{\text {diff } \underline{v}}$ in $O(N)$ operations.

For the matrix $\mathbf{C}$ we use wavelet compression: for finite intensity jump processes we have $Y<0, \sigma>0$. Smoothness of $k(z)$ for $z \neq 0$ causes rapid decay of the matrix elements $C_{(l, j),\left(l^{\prime}, j^{\prime}\right)}=c\left(\psi_{j}^{l}, \psi_{j^{\prime}}^{l^{\prime}}\right)$ as the levels $l, l^{\prime}$ increase. We can exploit this behavior and replace matrix elements for certain large values of $\left(l, l^{\prime}\right)$ with zero: for $\beta \in(0,1)$ we define the matrix $\tilde{\mathbf{C}}$ and the corresponding bilinear form $\tilde{c}$ by

$$
\tilde{C}_{(j, l),\left(j^{\prime}, l^{\prime}\right)}:= \begin{cases}C_{(j, l),\left(j^{\prime}, l^{\prime}\right)} & \text { if } l+l^{\prime} \leq \beta L \\ 0 & \text { otherwise }\end{cases}
$$

Proposition 5.3. Assume $Y \leq 2 \rho-2(p+1)$ and $\beta$ in (5.17) given by

$$
\beta=\frac{2 p+2-\rho}{\rho+\min \{-Y, 2 p+2\}} .
$$

Then the consistency condition

$$
\left|c\left(u_{h}, v_{h}\right)-\tilde{c}\left(u_{h}, v_{h}\right)\right| \leq C h^{p+1-\rho / 2}|\log h|^{\nu}\left\|u_{h}\right\|_{p+1}\left\|v_{h}\right\|_{\tilde{H}^{\rho / 2}}
$$

holds, and the number of nonzero matrix entries in $\tilde{\mathbf{C}}$ is bounded by $O\left(N^{\beta} \log N\right)$ with $\beta \leq 1$.

Proof. Let $u, v \in V_{h}$. For $u=\sum_{l=0}^{L} \sum_{j=1}^{M^{l}} u_{j}^{l} \psi_{j}^{l}$ we define $u^{l}:=\sum_{j=1}^{M^{l}} u_{j}^{l} \psi_{j}^{l}$, and analogously for $v$. The approximation property of $V^{l}$ implies that $\left\|u^{l}\right\|_{s} \leq C\left\|u^{l}\right\|_{s}$ for $-(p+1) \leq s \leq 0$ [14]. We then have with $\alpha:=\max \{Y / 2,-(p+1)\}, s:=p+1-\rho / 2, \delta:=\rho / 2-\alpha$

$$
\left|c\left(u^{l}, v^{l^{\prime}}\right)\right| \leq C\left\|u^{l}\right\|_{\tilde{H}^{\rho / 2}\left(\Omega_{R}\right)}\left\|v^{l^{\prime}}\right\|_{\tilde{H}^{\rho / 2}\left(\Omega_{R}\right)} \leq C^{\prime}\left\|u^{l}\right\|\left\|_{\alpha}\right\| v^{l^{\prime}}\left\|_{\alpha}=C^{\prime} 2^{-s l-\delta\left(l+l^{\prime}\right)}\right\| u^{l}\left\|_{p+1}\right\| v^{l^{\prime}} \|_{\rho / 2} .
$$

Therefore

$$
\begin{aligned}
|c(u, v)-\tilde{c}(u, v)| & \leq \sum_{\substack{l, l^{\prime}=0, \ldots, L \\
l+l^{\prime}>\beta L}}\left|c\left(u^{l}, v^{l^{\prime}}\right)\right| \leq C \sum_{\substack{l, l^{\prime}=0, \ldots, L \\
l+l^{\prime}>\beta L}} 2^{-s l-\delta\left(l+l^{\prime}\right)}\left\|u^{l}\right\|\left\|_{p+1}\right\| v^{l^{\prime}} \|_{\rho / 2} \\
& =\sum_{\substack{l, l^{\prime}=0, \ldots, L\\
}} Q_{l, l^{\prime}}\left\|u^{l}\right\|_{p+1}\left\|v^{l^{\prime}}\right\|_{\rho / 2} \leq\|Q\|_{2}\|u\|_{p+1}\|v\|_{\rho / 2} .
\end{aligned}
$$

Here $Q$ is the matrix with $Q_{l, l^{\prime}}=2^{-s l-\delta\left(l+l^{\prime}\right)}$ for $l+l^{\prime}>\beta L$ and $Q_{l, l^{\prime}}=0$ otherwise. Note that we have $\beta=s / \delta \leq 1$ by our assumption on $Y$. For $l+l^{\prime} \geq \beta L$ we have $s l+\delta\left(l+l^{\prime}\right) \geq s l+s L$. Hence we have 
$Q_{l, l^{\prime}} \leq 2^{-s L}$, and using geometric series we get $\|Q\|_{2} \leq\|Q\|_{1}^{1 / 2}\|Q\|_{\infty}^{1 / 2} \leq C 2^{-s L}=C h^{s}$. This proves the consistency condition (5.19). The number of nonzero matrix elements is

$$
\sum_{\substack{l, l^{\prime}=0, \ldots, L \\ l+l^{\prime} \leq \beta L}} 2^{l+l^{\prime}}=\sum_{k=0}^{\beta L} k 2^{k} \leq C L 2^{\beta L}
$$

For the compressed matrix $\tilde{\mathbf{C}}$ the operation $\underline{v} \mapsto \tilde{\mathbf{C}} \underline{v}$ can therefore be performed in $O\left(N^{\beta^{\prime}}\right)$ operations with $\beta^{\prime}<1$, and for large $N$ the work for matrix $\tilde{\mathbf{C}}$ becomes negligible compared with the work for the bandmatrix $\mathbf{A}_{\text {diff }}$.

We now consider the case of $p=1$ with piecewise linear functions. For $Y \geq 0$ we use the compression (5.11) with (5.14). For $\sigma>0$ and $Y<0$ we use the compression (5.17) with (5.18). In the case of a smooth kernel such as the Merton model from Section 3.3.1 we can use any negative $Y$ in (5.18) and obtain a compressed matrix $\tilde{\mathbf{C}}$ with $O\left(N^{1 / 3} \log N\right)$ nonzero elements and, by (5.16), a corresponding perturbed bilinear form $\tilde{a}_{R}$. In the case of Kou's model from Section 3.3.1 we obtain with $Y=-2$ a matrix $\tilde{\mathbf{C}}$ with $O\left(N^{1 / 2} \log N\right)$ nonzero elements.

\subsubsection{Perturbed $\theta$-scheme}

Using $\tilde{a}_{R}(\cdot, \cdot)$ in place of $a_{R}(\cdot, \cdot)$ in (5.9) gives perturbed $\theta$-schemes

$$
\begin{gathered}
\widetilde{U}_{h}^{0}=0 \\
\left(\frac{\widetilde{U}_{h}^{m+1}-\widetilde{U}_{h}^{m}}{k}, v_{h}\right)+\widetilde{a}_{R}\left(\widetilde{U}_{h}^{m+\theta}, v_{h}\right)=\left\langle f, v_{h}\right\rangle_{V^{*} \times V}
\end{gathered}
$$

for $m=0,1,2, \ldots, M-1$ and every $v_{h} \in V_{h}$, where again $\widetilde{U}_{h}^{m+\theta}:=\theta \widetilde{U}_{h}^{m+1}+(1-\theta) \widetilde{U}_{h}^{m}$. In matrix form, $(5.21 \mathrm{~b})$ reads

$$
\left(k^{-1} \mathbf{M}+\theta \tilde{\mathbf{A}}\right) \underline{\tilde{U}}^{m+1}=k^{-1} \mathbf{M} \underline{\tilde{U}}^{m}-(1-\theta) \tilde{\tilde{\mathbf{A}}} \underline{\tilde{U}}^{m}+\underline{f}, \quad m=0,1, \ldots, M-1
$$

where $\underline{\tilde{U}}^{m}$ is the coefficient vector of $\tilde{U}_{h}^{m}$ with respect to a basis of $V_{h}$.

\subsection{Convergence}

Consider now the sequence $\left\{\widetilde{U}_{h}^{m}\right\}_{m=0}^{M}$ of solutions to the perturbed $\theta$-scheme (5.21a), (5.21b). These solutions are stable and converge with optimal order as $h \rightarrow 0$, regardless of the wavelet compression.

We define for $v_{h} \in V_{h}$ and $f \in V_{h}^{*}$

$$
\left\|v_{h}\right\|_{\tilde{a}}:=\left(\tilde{a}_{R}\left(v_{h}, v_{h}\right)\right)^{1 / 2}, \quad\|f\|_{\tilde{\kappa}}:=\sup _{v_{h} \in V_{h}} \frac{\left(f, v_{h}\right)}{\left\|v_{h}\right\|_{\tilde{a}}}, \quad \lambda_{\tilde{A}}:=\sup _{v_{h} \in V_{h}} \frac{\left\|v_{h}\right\|^{2}}{\left\|v_{h}\right\|_{\tilde{*}}^{2}} .
$$

Theorem 5.4. Assume that the conditions (5.13), (5.15) hold. In the case of $0 \leq \theta<\frac{1}{2}$ assume the time-step restriction

$$
k<\frac{2}{(1-2 \theta) \lambda_{\tilde{A}}} .
$$

Assume that the exact solution $U_{R}(\tau, x)$ of (4.1)-(4.3) is sufficiently smooth. Then there holds the error estimate

$$
\left\|U_{R}(T, \cdot)-\widetilde{U}_{h}^{M}\right\|^{2}+k \sum_{m=0}^{M-1}\left\|U_{R}((m+\theta) k, \cdot)-\widetilde{U}_{h}^{m+\theta}\right\|_{a}^{2} \leq C\left(h^{2(p+1-\rho / 2)}|\log h|^{2 \nu+1}+k^{2 \mu}\right),
$$


where $C>0$ depends on $R, \nu$ is as in (5.15), $\mu=1$ if $\theta \neq \frac{1}{2}$ and $\mu=2$ otherwise.

The convergence result (5.24) is proved in [41], Theorem 5.4.

Remark 5.5. We can estimate $\lambda_{\tilde{A}}$ in (5.22) as follows: for $v_{h} \in V_{h}$ we have from the inverse inequality $\left\|w_{h}\right\|_{\tilde{a}} \leq C\left\|w_{h}\right\|_{V} \leq h^{-\rho / 2}\left\|w_{h}\right\|$ that

$$
\left\|v_{h}\right\|_{\tilde{*}}=\sup _{w_{h} \in V_{h}} \frac{\left(v_{h}, w_{h}\right)}{\left\|w_{h}\right\|_{\tilde{a}}} \geq C h^{\rho / 2} \sup _{w_{h} \in V_{h}} \frac{\left(v_{h}, w_{h}\right)}{\left\|w_{h}\right\|}=C h^{\rho / 2}\left\|v_{h}\right\|
$$

and therefore

$$
\lambda_{\tilde{A}}^{1 / 2}=\sup _{v_{h} \in V_{h}} \frac{\left\|v_{h}\right\|}{\left\|v_{h}\right\|_{\tilde{*}}} \leq C h^{-\rho / 2} .
$$

Hence there exists a positive constant $C_{*}$ independent of $h$ and $\theta$ such that the time-step restriction

$$
k \leq C_{*} \frac{h^{\rho}}{1-2 \theta}
$$

is sufficient for stability. For $\sigma>0$ and $\theta=0$ this gives to the well-known time-step restriction $k \leq C_{\theta} h^{2}$ for explicit schemes. Note that this restriction for $\theta=0$ is less severe for $\sigma=0$ and small values of $Y$.

\subsection{Approximate solution of linear equations and complexity}

In order to compute the approximate solution $\tilde{U}_{h}^{m}$ in $(5.21)$ for $m=1, \ldots, M$ we proceed as follows:

We first compute the mass matrix $\mathbf{M}$ in the wavelet basis with elements $M_{(l, j),\left(l^{\prime}, j^{\prime}\right)}$ where $O(N \log N)$ elements are nonzero.

Then we compute the compressed stiffness matrix $\tilde{\mathbf{A}}$ where $O(N(\log N))$ elements are nonzero, see Proposition 5.2. If explicit antiderivatives of the kernel function are available (as is often the case), the total cost for computing the stiffness matrix $\tilde{\mathbf{A}}$ is $O(N(\log N))$ operations. In other cases quadratures can be used. This preserves the consistency conditions $(5.13),(5.15)$ and the total cost of computing $\tilde{\mathbf{A}}$ is $O\left(N(\log N)^{2}\right)$.

For each time step we have to solve $(5.21 \mathrm{~b})$ : we have to find $\tilde{w}_{h}^{m}:=\tilde{U}_{h}^{m+1}-\tilde{U}_{h}^{m} \in V_{h}$ satisfying

$$
k^{-1}\left(\tilde{w}_{h}^{m}, v_{h}\right)+\theta \tilde{a}_{R}\left(\tilde{w}_{h}^{m}, v_{h}\right)=\left(f^{m+\theta}, v_{h}\right)-\tilde{a}_{R}\left(\tilde{U}_{h}^{m}, v_{h}\right) \quad \forall v_{h} \in V_{h}
$$

and then update $\tilde{U}_{h}^{m+1}:=\tilde{U}_{h}^{m}+\tilde{w}_{h}^{m}$. Let $\underline{\tilde{w}}^{m} \in \mathbb{R}^{N}$ denote the coefficient vectors of $\tilde{w}_{h}^{m}$ with respect to the wavelet basis, and $\mathbf{M}, \tilde{\mathbf{A}} \in \mathbb{R}^{N \times N}$ the mass and stiffness matrices corresponding to $(\cdot, \cdot)$ and $\tilde{a}_{R}(\cdot, \cdot)$ in this basis. Then we obtain for $\underline{\tilde{w}}^{m}$ a linear system $\mathbf{B} \underline{\tilde{w}}^{m}=\underline{\tilde{b}}^{m}$ with the matrix $\mathbf{B}=k^{-1} \mathbf{M}+\theta \tilde{\mathbf{A}}$ and a known right-hand side vector $\underline{\tilde{b}}^{m}$.

For a standard finite element basis the matrix $\mathbf{B}$ has a condition number of order $h^{-\rho}$ for small $h$ and fixed $k$. For the matrix $\mathbf{B}$ in the wavelet basis we can achieve a uniformly bounded condition number if we scale the rows and columns of $\mathbf{B}$ as follows: let $\mu_{l}:=\left(k^{-1}+\theta 2^{\rho l}\right)^{1 / 2}$ and let $\hat{B}_{(l, j),\left(l^{\prime}, j^{\prime}\right)}:=\mu_{l}^{-1} \mu_{l^{\prime}}^{-1} B_{(l, j),\left(l^{\prime}, j^{\prime}\right)}$. Let in what follows $\|\cdot\|$ denote the 2 -norm of a vector, or the 2 -norm of a matrix.

Let $\mathbf{D}$ denote the diagonal matrix with entries $D_{(l, j),(l, j)}=2^{l \rho / 2}$. Scaling with the diagonal matrix $\mathbf{S}:=$ $\left(k^{-1} \mathbf{I}+\theta \mathbf{D}^{2}\right)^{1 / 2}$ yields with $\hat{\mathbf{B}}=\mathbf{S}^{-1} \mathbf{B} \mathbf{S}^{-1}$

$$
\lambda_{\min }\left(\left(\hat{\mathbf{B}}+\hat{\mathbf{B}}^{\top}\right) / 2\right) \geq C_{1}, \quad\|\hat{\mathbf{B}}\| \leq C_{2}
$$

for some $C_{1}, C_{2}>0$ independent of $h$ and $k$. This implies that a step of the GMRES method for the solution of a linear system with matrix $\hat{\mathbf{B}}$ has a convergence factor $\leq q<1$ independent of $L$ (see [41]). 
Remark 5.6. If one considers operators $\mathcal{A}$ with values of $\sigma$ tending to zero, the convergence factor will not stay uniformly bounded by $q<1$. One can obtain a uniformly bounded convergence factor if one modifies the scaling by using $\mu_{l}:=\left(k^{-1}+\theta\left[\sigma 2^{2 l}+C 2^{Y l}\right]\right)^{1 / 2}$. This follows from Proposition 4.2: because of $(4.9)$ we can assume $c_{2}=0$ and have that $a_{R}(u, u) \sim C\|u\|_{\tilde{H}^{Y / 2}\left(\Omega_{R}\right)}^{2}+\sigma^{2}|u|_{1}^{2}$. Then by (5.10) the weight $2^{2 l}$ gives a norm equivalent to $\|\cdot\|_{1}$, and the weight $2^{Y l}$ gives a norm equivalent to $\|\cdot\|_{\tilde{H}^{Y / 2}\left(\Omega_{R}\right)}$.

For a function $v_{h} \in V_{h}$ with coefficient vector $\underline{v}$ and scaled coefficient vector $\underline{\hat{v}}=\mathbf{S} \underline{v}$ we have that with $b(u, v):=k^{-1}(u, v)+\theta \tilde{a}_{R}(u, v)$ and $\|v\|_{b}^{2}:=b(v, v)$

$$
\|\underline{\hat{v}}\|^{2} \sim \underline{\hat{v}}^{\top} \hat{\mathbf{B}} \underline{\hat{v}}=\left\|v_{h}\right\|_{b}^{2} .
$$

A functional $g_{h} \in V_{h}^{*}$ corresponds to a coefficient vector $\underline{g}$ so that $\left(g_{h}, v_{h}\right)=\underline{g}^{\top} \underline{v}$, and a scaled vector $\underline{\hat{g}}=\mathbf{S}^{-1} \underline{g}$ so that $\left(g_{h}, v_{h}\right)=\underline{\hat{g}}^{\top} \underline{\hat{v}}$.

We now define the perturbed $\theta$-scheme with GMRES approximation as follows: pick a value $m_{0} \geq 1$ for the restart number, e.g., $m_{0}=1$, and a value $n_{G}$ for the number of iterations. At each time step we want to find an approximation of $w_{h, *}^{m}$ satisfying

$$
b\left(w_{h, *}^{m}, v_{h}\right)=\left(f^{m+\theta}, v_{h}\right)-\tilde{a}_{R}\left(\check{U}_{h}^{m}, v_{h}\right) \quad \text { for all } v_{h} \in V_{h}, \quad \check{U}_{h}^{0}=0
$$

which corresponds to a scaled linear system $\hat{\mathbf{B}} \underline{\hat{w}}_{*}^{m}=\underline{\hat{b}}^{m}$. We solve this system approximately with $n_{G}$ steps of $\operatorname{GMRES}\left(m_{0}\right)$, using zero as initial guess, yielding an approximation $\underline{\hat{w}}^{m}$ of the exact solution $\underline{\hat{w}}_{*}^{m}$. We then let $\check{U}_{h}^{m+1}:=\check{U}_{h}^{m}+w_{h}^{m}$, where $w_{h}^{m} \in V_{h}$ is the function corresponding to the scaled vector $\underline{\hat{w}}^{m}$. Then we have, see Theorem 6.3 in [41].

Theorem 5.7. Assume that the consistency conditions (5.13), (5.15) hold. For $\theta \in\left[0, \frac{1}{2}\right)$ assume $\sigma:=k(1-$ $2 \theta) \lambda_{A}<2$. Then the solution $\check{U}_{h}^{m}$ of the $\theta$-scheme with wavelet compression and approximate GMRES solution satisfies the same error bound as $\tilde{U}_{h}^{m}$ in (5.24) if $n_{G} \geq C|\log h|$. Given the compressed stiffness matrix $\tilde{\mathbf{A}}$, the work for computing $\check{U}_{h}^{1}, \ldots, \check{U}_{h}^{M}$ is bounded by $C M N(\log N)^{2}$ floating point operations.

\subsection{Numerical results}

We restrict the numerical experiments to vanishing interest rate, i.e., $r=0$. In Figure 3 we present the option prices versus the stock price $S$ for the case of an European call contract on Lévy driven assets. We use different maturities (top) and different strike prices $K$ (bottom) for an extended CGMY process [12] with $\sigma=0.1, C=1, G=1.8, M=2.5$ and $Y=0.2$. We plot for each case (top right and top bottom, respectively) the difference between the option prices in the jump-diffusion case and the prices obtained by the standard Black-Scholes formula (only diffusion) with $\sigma=0.1$.

In Figure 4 we plot the option prices versus the stock price $S$ for the case of an European call contract on pure jump Lévy driven assets $(\sigma=0)$ at different maturities (left and right (zoom)); CGMY parameters are: $Y=0.1430, C=9.61, G=9.97$ and $M=16.51$ (see [12]). Note that in our theoretical analysis we assume that one removes for $Y<1$ the drift term with the transformation in Section 4.4. In our numerical experiments we used the original equations without this transformation, but the results still appear to be stable.

In the next set of numerical experiments we consider the variance gamma process. It is a particular case of the CGMY process with $Y=0$. Here explicit formulas for the prices of European options are available [28]. The parameters are here $Y=0, G=2.78, M=2.86$ and $C=1.0$. In Figure 5 we compare our numerical results obtained with the exact VG prices obtained by the explicit formulae in [28] for different strike prices $K$ and maturity $T=0.5$. The computed values are on top of the exact prices obtained by the explicit formula in [28]. Note that our theoretical results only apply to the case $Y>0$, however even in the limiting case $Y=0$ the numerical method appears to be accurate. 

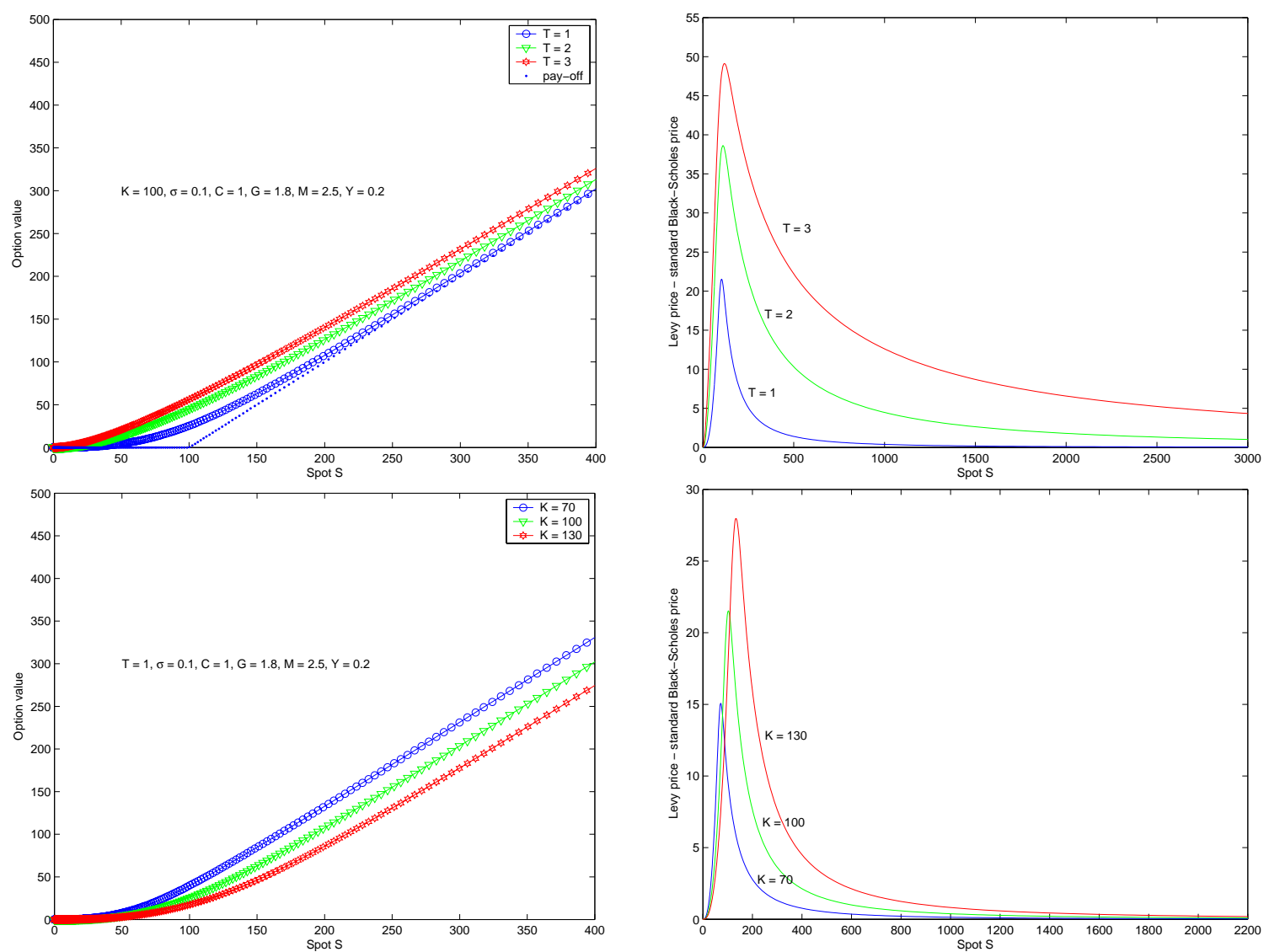

Figure 3. Option prices versus the stock price $S$ for the case of an European call contract on Lévy driven assets as compared with the Black-Scholes prices; different maturities (top) and different strike prices $K$ (bottom) for the case of an extended CGMY process with $\sigma=0.1$, $Y=0.2, G=1.8$ and $M=2.5$.
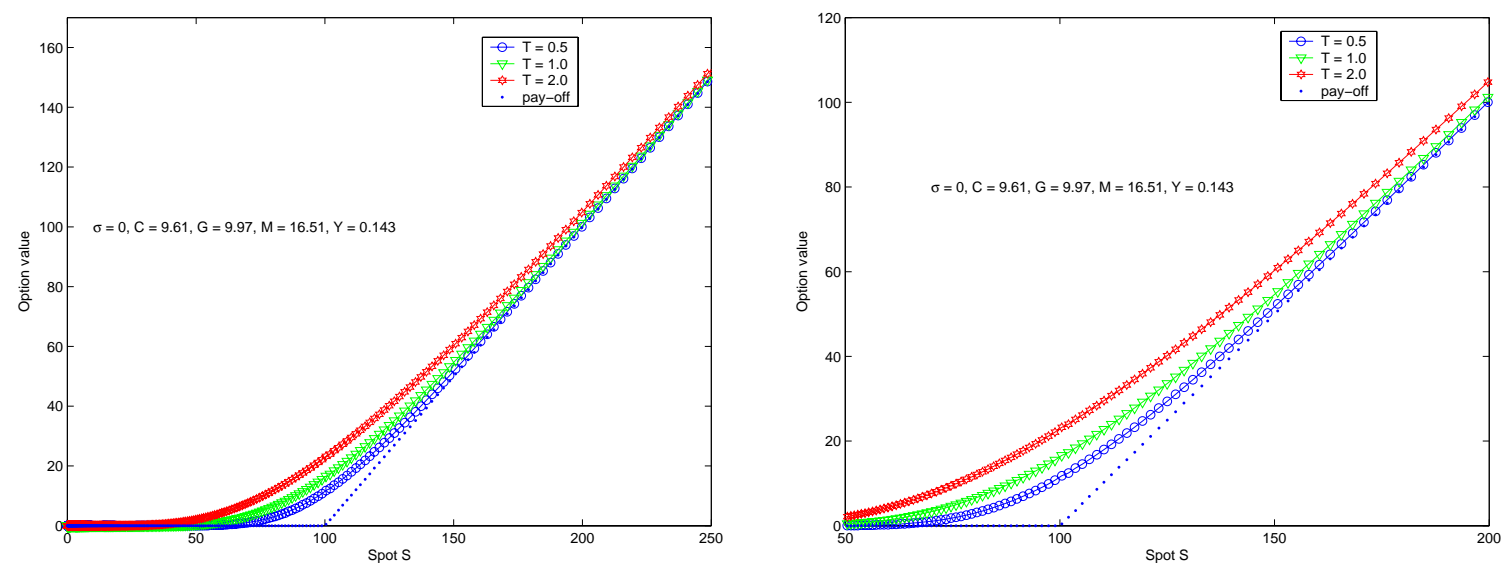

FigurE 4. Option prices versus the stock price $S$ for the case of an European call contract on pure jump Lévy driven assets $(\sigma=0)$ at different maturities (left and right (zoom)); CGMY parameters are: $Y=0.1430, C=9.61, G=9.97$ and $M=16.51$. 


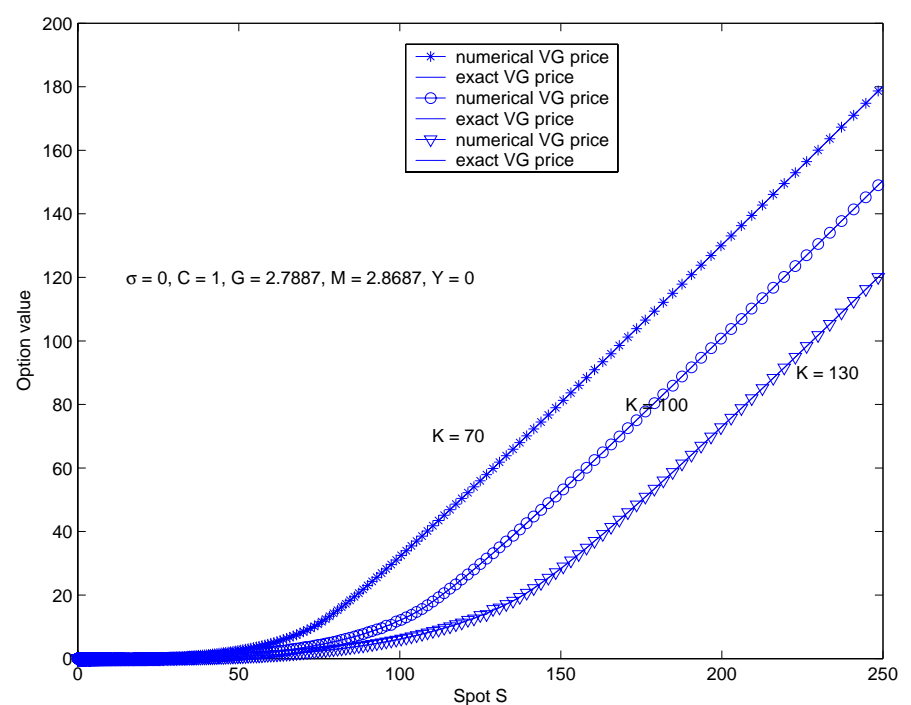

Figure 5. Option prices versus the stock price $S$ for the case of an European call contract on VG (pure jump) driven assets for different strike prices $K$ at maturity $T=0.5$; VG parameters: $\sigma_{V G}=0.5, \nu_{V G}=1.0, \theta_{V G}=-0.01 \Rightarrow$ CGMY parameters: $Y=0, G=2.78$ and $M=2.86$.

Figure 6 shows pricing of options with the forward Euler scheme, i.e. with $\theta=0$. We clearly see the impact of the CFL-condition (5.25) - if it is violated, instability results. In the jump-diffusion case, the time-step restriction (5.25) renders the explicit scheme inefficient. In the pure jump case, however, CFL-condition (5.25) yields a competitive scheme for $Y \leq 1$; again the condition (5.25) is sharp, as is evidenced by Figure 7 right and Figure 8.

\section{A. Proof of Theorem 3.4}

We consider $\varphi, \psi \in C_{0}^{\infty}(\mathbb{R})$. Integration by parts and $r=0$ in $a^{-\eta}$ in (3.22) gives

$$
\begin{aligned}
a^{-\eta}(\varphi, \psi)= & -\frac{\sigma^{2}}{2} \int_{\mathbb{R}}\left(\frac{\mathrm{d}^{2} \varphi}{\mathrm{d} x^{2}}(x)-\frac{\mathrm{d} \varphi}{\mathrm{d} x}(x)\right) \psi(x) \mathrm{e}^{-2 \eta(x)} \mathrm{d} x \\
& -\int_{\mathbb{R}} \int_{\mathbb{R}}\left\{\varphi(x+y)-\varphi(x)-y \frac{\mathrm{d} \varphi}{\mathrm{d} x}(x) \chi_{\{|y| \leq 1\}}(y)\right\} k(y) \psi(x) \mathrm{e}^{-2 \eta(x)} \mathrm{d} y \mathrm{~d} x \\
& +c_{\exp } \int_{\mathbb{R}} \frac{\mathrm{d} \varphi}{\mathrm{d} x}(x) \psi(x) \mathrm{e}^{-2 \eta(x)} \mathrm{d} x \\
= & \frac{\sigma^{2}}{2} \int_{\mathbb{R}} \frac{\mathrm{d} \varphi}{\mathrm{d} x} \frac{d \psi}{\mathrm{d} x}(x) \mathrm{e}^{-2 \eta(x)} \mathrm{d} x+\frac{\sigma^{2}}{2} \int_{\mathbb{R}} \frac{\mathrm{d} \varphi}{\mathrm{d} x}(x) \psi(x)\left(-2 \eta^{\prime}(x)+1\right) \mathrm{e}^{-2 \eta(x)} \mathrm{d} x \\
& -\int_{\mathbb{R}} \int_{\mathbb{R}} \int_{0}^{1} \mathrm{~d} \theta \frac{\mathrm{d} \varphi}{\mathrm{d} x}(x+\theta y) y \chi_{\{|y| \geq 1\}}(y) k(y) \psi(x) \mathrm{e}^{-2 \eta(x)} \mathrm{d} x \mathrm{~d} y \\
& -\int_{\mathbb{R}} \int_{\mathbb{R}} \int_{0}^{1} \mathrm{~d} \theta \int_{0}^{\theta} \mathrm{d} \theta^{\prime} \frac{\mathrm{d}^{2} \varphi}{\mathrm{d} x^{2}}\left(x+\theta^{\prime} y\right) y^{2} \chi_{\{|y| \leq 1\}}(y) k(y) \psi(x) \mathrm{e}^{-2 \eta(x)} \mathrm{d} x \mathrm{~d} y \\
& +c_{\exp } \int_{\mathbb{R}} \frac{\mathrm{d} \varphi}{\mathrm{d} x}(x) \psi(x) \mathrm{e}^{-2 \eta(x)} \mathrm{d} x .
\end{aligned}
$$



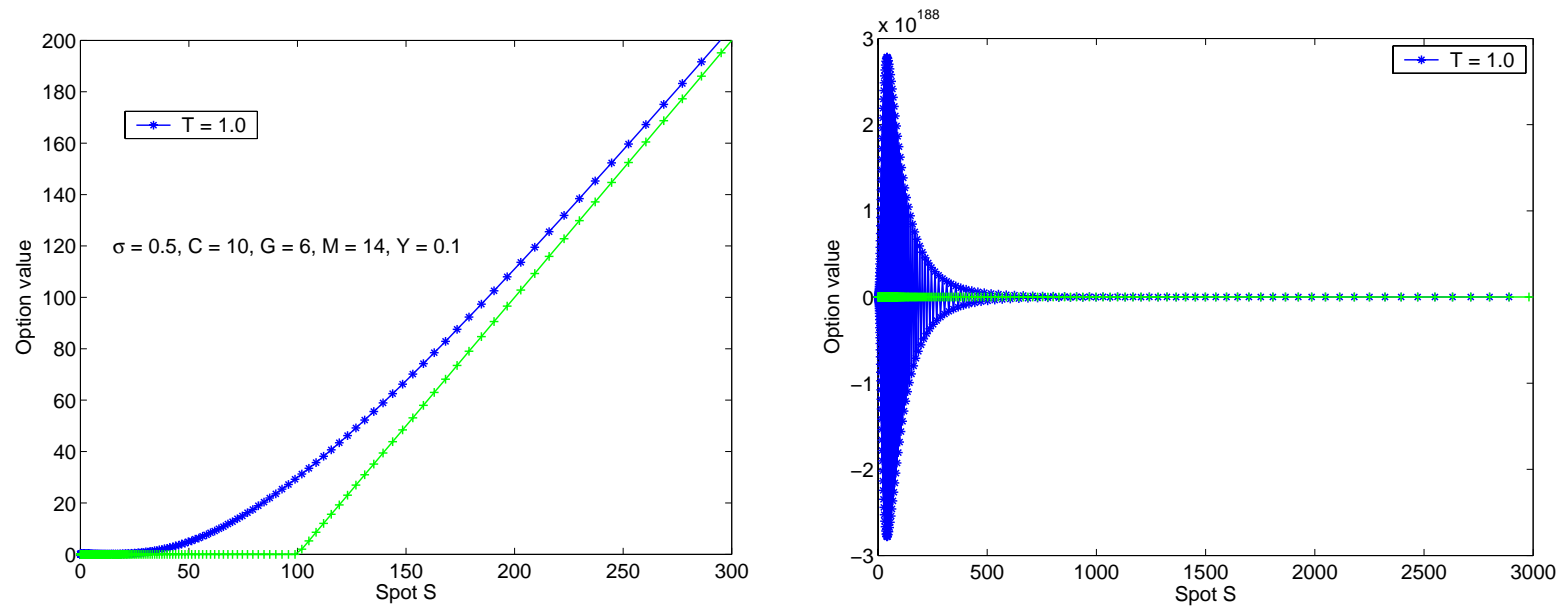

Figure 6. Explicit Euler scheme $(\theta=0.0), h=0.0312(L=8, R=8), T=1.0$; CGMY parameters: $C=10.0, G=6.0, M=14.0, Y=0.1, \sigma=0.5$; stable $k=h^{2}$ (left), unstable: $k=2 h^{2}$.
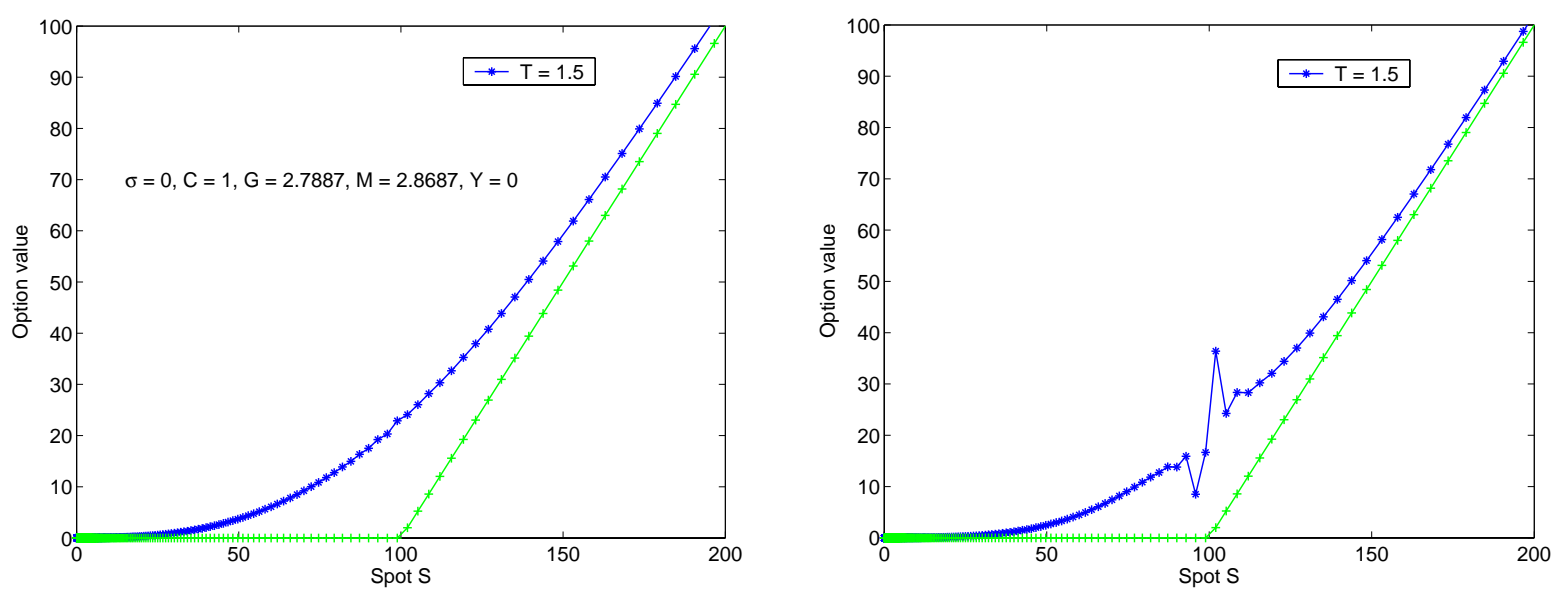

Figure 7. Explicit Euler scheme, pure jump VG process: $\theta=0.0, L=8 ; \alpha$ is the coefficient in the convection term $\alpha \frac{\partial u}{\partial x}$. $|\alpha| \frac{k}{h} \leq 1$ stable; $|\alpha| \frac{k}{h}>1$ unstable VG parameters: $\sigma_{V G}=0.5$, $\nu_{V G}=1.0, \theta_{V G}=-0.01 \Rightarrow$ CGMY parameters: $Y=0, G=2.78$ and $M=2.86$.

We write therefore $a^{-\eta}(\varphi, \psi)=a_{1}^{-\eta}(\varphi, \psi)+a_{2}^{-\eta}(\varphi, \psi)+a_{3}^{-\eta}(\varphi, \psi)$, where

$$
\begin{aligned}
a_{1}^{-\eta}(\varphi, \psi)= & \frac{\sigma^{2}}{2} \int_{\mathbb{R}} \frac{\mathrm{d} \varphi}{\mathrm{d} x} \frac{\mathrm{d} \psi}{\mathrm{d} x}(x) \mathrm{e}^{-2 \eta(x)} \mathrm{d} x+\frac{\sigma^{2}}{2} \int_{\mathbb{R}} \frac{\mathrm{d} \varphi}{\mathrm{d} x}(x) \psi(x)\left(-2 \eta^{\prime}(x)+1\right) \mathrm{e}^{-2 \eta(x)} \mathrm{d} x, \\
a_{2}^{-\eta}(\varphi, \psi)= & -\int_{\mathbb{R}} \int_{\mathbb{R}} \int_{0}^{1} \mathrm{~d} \theta \frac{\mathrm{d} \varphi}{\mathrm{d} x}(x+\theta y) y \chi_{\{|y| \geq 1\}}(y) k(y) \psi(x) \mathrm{e}^{-2 \eta(x)} \mathrm{d} x \mathrm{~d} y, \\
a_{3}^{-\eta}(\varphi, \psi)= & -\int_{\mathbb{R}} \int_{\mathbb{R}} \int_{0}^{1} \mathrm{~d} \theta \int_{0}^{\theta} \mathrm{d} \theta^{\prime} \frac{\mathrm{d}^{2} \varphi}{\mathrm{d} x^{2}}\left(x+\theta^{\prime} y\right) y^{2} \chi_{\{|y| \leq 1\}}(y) k(y) \psi(x) \mathrm{e}^{-2 \eta(x)} \mathrm{d} x \mathrm{~d} y \\
& +c_{\exp } \int_{\mathbb{R}} \frac{\mathrm{d} \varphi}{\mathrm{d} x}(x) \psi(x) \mathrm{e}^{-2 \eta(x)} \mathrm{d} x .
\end{aligned}
$$



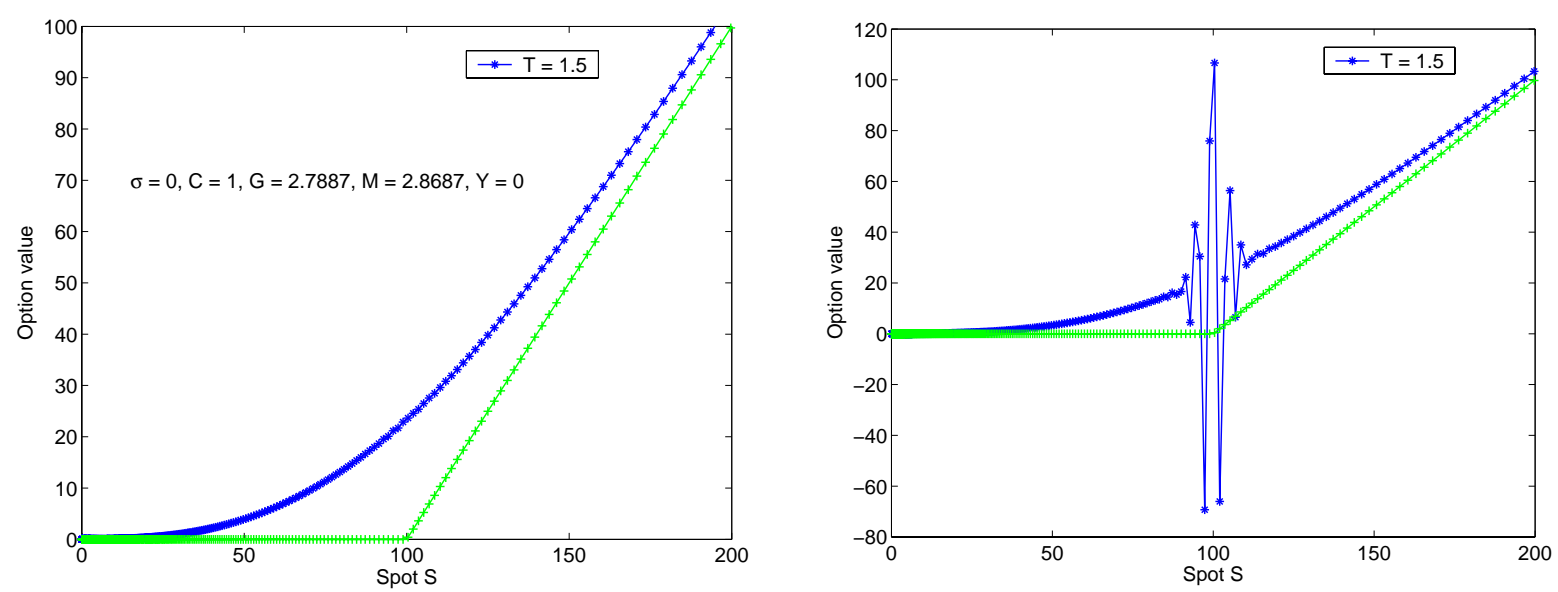

FIGURE 8. Explicit Euler scheme, pure jump VG process: $\theta=0.0, L=9 ;|\alpha| \frac{k}{h} \leq 1$ (left) stable, $|\alpha| \frac{k}{h}>1$ (right) unstable; VG parameters: $\sigma_{V G}=0.5, \nu_{V G}=1.0, \theta_{V G}=-0.01 \Rightarrow$ CGMY parameters: $Y=0, G=2.78$ and $M=2.86$.

We analyse each $a_{j}^{\eta}(\cdot, \cdot)(j=1,2,3)$ separately. For the bilinear form $a_{1}^{-\eta}$ we obtain

$$
\left|a_{1}^{-\eta}(\varphi, \psi)\right| \leq C_{1}\left(\left\|\eta^{\prime}\right\|_{L^{\infty}(\mathbb{R})}\right)\|\varphi\|_{H_{-\eta}^{1}(\mathbb{R})}\|\psi\|_{H_{-\eta}^{1}(\mathbb{R})}
$$

and

$$
\begin{aligned}
a_{1}^{-\eta}(\varphi, \varphi) & \geq \frac{\sigma^{2}}{2}\left\|\varphi^{\prime}\right\|_{L_{-\eta}^{2}(\mathbb{R})}^{2}-\frac{\sigma^{2}}{2}\left(2\left\|\eta^{\prime}\right\|_{L^{\infty}(\mathbb{R})}+1\right)\left\|\varphi^{\prime}\right\|_{L_{-\eta}^{2}(\mathbb{R})}\|\varphi\|_{L_{-\eta}^{2}(\mathbb{R})} \\
& \geq \frac{\sigma^{2}}{4}\left\|\varphi^{\prime}\right\|_{L_{-\eta}^{2}(\mathbb{R})}^{2}-c_{1}\left(\left\|\eta^{\prime}\right\|_{L^{\infty}(\mathbb{R})}\right)\|\varphi\|_{L_{-\eta}^{2}(\mathbb{R})}^{2} .
\end{aligned}
$$

In order to estimate $a_{2}^{-\eta}(\cdot, \cdot)$ we write it first in the following form

$$
a_{2}^{-\eta}(\varphi, \psi)=-\int_{\mathbb{R}} \int_{\mathbb{R}} \int_{0}^{1} \mathrm{~d} \theta \frac{\mathrm{d} \varphi}{\mathrm{d} x}(x+\theta y) \mathrm{e}^{-\eta(x+\theta y)} \mathrm{e}^{\eta(x+\theta y)-\eta(x)} y \chi_{\{|y| \geq 1\}}(y) k(y) \psi(x) \mathrm{e}^{-\eta(x)} \mathrm{d} x \mathrm{~d} y .
$$

By (3.24) we obtain

$$
\left|a_{2}^{-\eta}(\varphi, \psi)\right| \leq \int_{\mathbb{R}} \mathrm{e}^{\eta(y)}|y| \chi_{\{|y| \geq 1\}}(y) k(y) \mathrm{d} y \cdot\left\|\phi^{\prime}\right\|_{L_{-\eta}^{2}(\mathbb{R})}\|\psi\|_{L_{-\eta}^{2}(\mathbb{R})} .
$$

Hence, since $C(\eta):=\int_{\mathbb{R}} \mathrm{e}^{\eta(y)}|y| \chi_{\{|y| \geq 1\}}(y) k(y) \mathrm{d} y<\infty$ by assumption (3.25), $a_{2}^{-\eta}(\cdot, \cdot)$ is a bounded bilinear form on $H_{-\eta}^{1}(\mathbb{R}) \times H_{-\eta}^{1}(\mathbb{R})$.

Finally, we analyse $a_{3}^{-\eta}(\cdot, \cdot)$ and we show that for all $\varepsilon>0$ there exists $C_{\varepsilon}>0$ such that

$$
\left|a_{3}^{-\eta}(\varphi, \psi)\right| \leq\|\varphi\|_{H_{-\eta}^{1}(\mathbb{R})}\left(\varepsilon\|\psi\|_{H_{-\eta}^{1}(\mathbb{R})}+C_{\varepsilon}\|\psi\|_{L_{-\eta}^{2}(\mathbb{R})}\right) .
$$


To prove (A.3), let $\delta \in(0,1)$ be arbitrary, but fixed. We start by writing $a_{3}^{-\eta}(\cdot, \cdot)$ in the following form:

$$
\begin{aligned}
a_{3}^{-\eta}(\varphi, \psi)= & -\int_{\mathbb{R}} \int_{\mathbb{R}} \int_{0}^{1} \mathrm{~d} \theta \int_{0}^{\theta} \mathrm{d} \theta^{\prime} \frac{\mathrm{d}^{2} \varphi}{\mathrm{d} x^{2}}\left(x+\theta^{\prime} y\right) y^{2} \chi_{\{|y| \leq \delta\}}(y) k(y) \psi(x) \mathrm{e}^{-2 \eta(x)} \mathrm{d} x \mathrm{~d} y \\
& -\int_{\mathbb{R}} \int_{\mathbb{R}} \int_{0}^{1} \mathrm{~d} \theta \frac{\mathrm{d} \varphi}{\mathrm{d} x}(x+\theta y) y \chi_{\{\delta \leq|y| \leq 1\}}(y) k(y) \psi(x) \mathrm{e}^{-2 \eta(x)} \mathrm{d} x \\
& +\int_{\mathbb{R}} \int_{\mathbb{R}} \frac{\mathrm{d} \varphi}{\mathrm{d} x}(x) \psi(x) y \chi_{\{\delta \leq|y| \leq 1\}}(y) k(y) \psi(x) \mathrm{e}^{-2 \eta(x)} \mathrm{d} x \\
& +c_{\exp } \int_{\mathbb{R}} \frac{\mathrm{d} \varphi}{\mathrm{d} x}(x) \psi(x) \mathrm{e}^{-2 \eta(x)} \mathrm{d} x \\
= & \int_{\mathbb{R}} \int_{\mathbb{R}} \int_{0}^{1} \mathrm{~d} \theta \int_{0}^{\theta} \mathrm{d} \theta^{\prime} \frac{\mathrm{d} \varphi}{\mathrm{d} x}\left(x+\theta^{\prime} y\right) \mathrm{e}^{-\eta\left(x+\theta^{\prime} y\right)} y^{2} \chi_{\{|y| \leq \delta\}}(y) k(y) . \\
& \cdot\left\{\frac{d \psi}{\mathrm{d} x}(x)-2 \eta^{\prime}(x) \psi(x)\right\} \mathrm{e}^{\eta\left(x+\theta^{\prime} y\right)-\eta(x)} \mathrm{e}^{-\eta(x)} \mathrm{d} x \mathrm{~d} y \\
& -\int_{\mathbb{R}} \int_{\mathbb{R}} \int_{0}^{1} \mathrm{~d} \theta \frac{\mathrm{d} \varphi}{\mathrm{d} x}(x+\theta y) \mathrm{e}^{-\eta(x+\theta y)} y \chi_{\{\delta \leq|y| \leq 1\}}(y) k(y) \mathrm{e}^{\eta(x+\theta y)-\eta(x)} \psi(x) \mathrm{e}^{-\eta(x)} \mathrm{d} x \mathrm{~d} y \\
& +\int_{\mathbb{R}} \int_{\mathbb{R}} \frac{\mathrm{d} \varphi}{\mathrm{d} x}(x) \psi(x) y \chi_{\{\delta \leq|y| \leq 1\}}(y) k(y) \psi(x) \mathrm{e}^{-2 \eta(x)} \mathrm{d} x \\
& +c_{\exp } \int_{\mathbb{R}} \frac{\mathrm{d} \varphi}{\mathrm{d} x}(x) \psi(x) \mathrm{e}^{-2 \eta(x)} \mathrm{d} x .
\end{aligned}
$$

$\operatorname{By}(3.24), \mathrm{e}^{\eta(x+\theta y)-\eta(x)} \leq \mathrm{e}^{\eta(y)} \leq C$ for all $|y| \leq 1$. We obtain therefore the following estimate

$$
\begin{aligned}
\left|a_{3}^{-\eta}(\varphi, \psi)\right| & \leq C_{3}\left(\left\|\eta^{\prime}\right\|_{L^{\infty}(\mathbb{R})}\right) \int_{\mathbb{R}} y^{2} \chi_{\{|y| \leq \delta\}}(y) k(y) \mathrm{d} y \cdot\|\varphi\|_{H_{-\eta}^{1}(\mathbb{R})}\|\psi\|_{H_{-\eta}^{1}(\mathbb{R})} \\
& +\left(C \int_{\mathbb{R}}|y| \chi_{\{\delta \leq|y| \leq 1\}}(y) k(y) \mathrm{d} y+\left|c_{\exp }\right|\right) \cdot\left\|\varphi^{\prime}\right\|_{L_{-\eta}^{2}(\mathbb{R})}\|\psi\|_{L_{-\eta}^{2}(\mathbb{R})} .
\end{aligned}
$$

Since by (3.3) $\int_{\mathbb{R}} y^{2} \chi_{\{|y| \leq \delta\}}(y) k(y) \mathrm{d} y \rightarrow 0$ as $\delta \rightarrow 0$, for $\varepsilon>0$ fixed we can choose $\delta=\delta(\varepsilon) \in(0,1)$ sufficiently small such that (A.3) holds.

The above calculations with $\eta$ replaced by $-\eta$ lead to identical conclusions, if instead of (3.24), (3.26) holds and if condition (3.25) is replaced by (3.27).

Remark A.1. (3.24) and (3.25) hold for all $\eta$ of the form

$$
\eta(x)=\left\{\begin{array}{l}
\nu_{1}|x| \text { if } x<0 \\
\nu_{2}|x| \text { if } x>0
\end{array}\right.
$$

with $0 \leq \nu_{1}<G$ and $0 \leq \nu_{2}<M$. We distinguish 4 cases

Case 1. $(x+\theta y>0, x>0)$. Then, $\left.\nu_{2}(x+\theta y)-\nu_{2} x=\nu_{2} \theta y \leq \mathrm{a}\right)-\nu_{1} y$, if $y<0$ b) $\nu_{2} y$, if $y>0$.

Case 2. $(x+\theta y>0, x<0)$. Here, $\nu_{2}(x+\theta y)+\nu_{1} x=\left(\nu_{1}+\nu_{2}\right) x+\nu_{2} \theta y \leq \nu_{2} \theta y \leq \nu_{2} y(=\eta(y)$, since $y>0$ in this case).

Case 3. $(x+\theta y<0, x>0)$. Then, $-\nu_{1}(x+\theta y)-\nu_{2} x=-\left(\nu_{1}+\nu_{2}\right) x-\nu_{1} \theta y \leq-\nu_{1} y(=\eta(y)$, since $y<0$ here).

Case 4. $(x+\theta y<0, x<0)$. Here, $\left.-\nu_{1}(x+\theta y)+\nu_{1} x=-\nu_{1} \theta y \leq \mathrm{a}\right)-\nu_{1} y$ if $y<0$ or b) $\nu_{2} y$ if $y>0$.

Acknowledgements. We thank Freddy Delbaen and Thorsten Rheinländer for many helpful discussions on Lévy processes, Dilip Madan for pointing out $[12,28]$ to us and Ali Hirsa for stimulating discussions and for providing a MATLAB implementation of the closed form solution for European VG. 


\section{REFERENCES}

[1] R.A. Adams, Sobolev Spaces. Academic Press, New York (1978).

[2] H. Amann, Linear and Quasilinear Parabolic Problems, Vol. I: Abstract Linear Theory, Monographs Math. Birkhäuser, Basel 89 (1995).

[3] O.E. Barndorff-Nielsen, Exponentially decreasing distributions for the logarithm of particle size. Proc. Roy. Soc. London A 353 (1977) 401-419.

[4] O.E. Barndorff-Nielsen, Normal inverse Gaussian distributions and stochastic volatility modelling. Scand. J. Statis. 24 (1997) $1-14$.

[5] O.E. Barndorff-Nielsen and N. Shepard, Non-Gaussian Ornstein-Uhlenbeck based models and some of their uses in financial economics. J. Roy. Stat. Soc. B 63 (2001) 167-241.

[6] A. Bensoussan and J.-L. Lions, Impulse control and quasi-variational inequalities. Gauthier-Villars, Paris (1984).

[7] J. Bertoin, Lévy processes. Cambridge University Press (1996).

[8] F. Black and M. Scholes, The Pricing of Options and Corporate Liabilities. J. Political Economy 81 (1973) 637-654.

[9] S. Boyarchenko and S. Levendorski, Barrier options and touch-and-out options under regular Lévy processes of exponential type. Ann. Appl. Probab. 12 (2002) 1261-1298.

[10] S. Boyarchenko and S. Levendorski, Option pricing for truncated Lévy processes. Int. J. Theor. Appl. Finance 3 (2000) 549-552.

[11] P. Carr and D. Madan, Option valuation using the FFT. J. Comp. Finance 2 (1999) 61-73.

[12] P. Carr, H. Geman, D.B. Madan and M. Yor, The fine structure of asset returns: an empirical investigation. J. Business $\mathbf{7 5}$ (2002) 305-332.

[13] T. Chan, Pricing contingent claims on stocks driven by Lévy processes. Ann. Appl. Probab. 9 (1999) 504-528.

[14] A. Cohen, Wavelet methods for operator equations, P.G. Ciarlet and J.L. Lions Eds., Elsevier, Amsterdam, Handb. Numer. Anal. VII (2000).

[15] R. Cont and P. Tankov, Financial modelling with jump processes. Chapman and Hall/CRC Press (2003).

[16] F. Delbaen and W. Schachermayer, The variance-optimal martingale measure for continuous processes. Bernoulli 2 (1996) 81-105.

[17] F. Delbaen, P. Grandits, T. Rheinländer, D. Samperi, M. Schweizer and C. Stricker, Exponential hedging and entropic penalties. Math. Finance 12 (2002) 99-123.

[18] E. Eberlein, Application of generalized hyperbolic Lévy motions to finance, in Lévy Processes: Theory and Applications, O.E. Barndorff-Nielsen, T. Mikosch and S. Resnick Eds., Birkhäuser (2001) 319-337.

[19] H. Föllmer and M. Schweizer, Hedging of contingent claims under incomplete information, in Applied Stochastic Analysis, M.H.A. Davis and R.J. Elliot Eds., Gordon and Breach New York (1991) 389-414.

[20] J. Jacod and A.N. Shiryaev, Limit Theorems for Stochastic Processes. Springer-Verlag, Berlin (1987).

[21] P. Jaillet, D. Lamberton and B. Lapeyre, Variational inequalities and the pricing of American options. Acta Appl. Math. 21 (1990) 263-289.

[22] R. Kangro and R. Nicolaides, Far field boundary conditions for Black-Scholes equations. SIAM J. Numer. Anal. 38 (2000) $1357-1368$.

[23] I. Karatzas and S.E. Shreve, Methods of Mathematical Finance. Springer-Verlag (1999).

[24] G. Kou, A jump diffusion model for option pricing. Mange. Sci. 48 (2002) 1086-1101.

[25] D. Lamberton and B. Lapeyre, Introduction to Stochastic Calculus Applied to Finance. Chapman \& Hall (1997).

[26] J.L. Lions and E. Magenes, Non-homogeneous boundary value problems and applications. Springer-Verlag, Berlin (1972).

[27] D.B. Madan and E. Seneta, The variance gamma (V.G.) model for share market returns. J. Business 63 (1990) 511-524.

[28] D.B. Madan, P. Carr and E. Chang, The variance gamma process and option pricing. Eur. Finance Rev. 2 (1998) 79-105.

[29] A.M. Matache, T. von Petersdorff and C. Schwab, Fast deterministic pricing of options on Lévy driven assets. Report 200211, Seminar for Applied Mathematics, ETH Zürich. http://www.sam.math.ethz.ch/reports/details/include.shtml?2002/ 2002-11.html

[30] A.M. Matache, P.A. Nitsche and C. Schwab, Wavelet Galerkin pricing of American options on Lévy driven assets. Research Report 2003-06, Seminar for Applied Mathematics, ETH Zürich, http://www.sam.math.ethz.ch/reports/ details/include.shtml?2003/2003-06.html

[31] R.C. Merton, Option pricing when the underlying stocks are discontinuous. J. Financ. Econ. 5 (1976) 125-144.

[32] D. Nualart and W. Schoutens, Backward stochastic differential equations and Feynman-Kac formula for Lévy processes, with applications in finance. Bernoulli 7 (2001) 761-776.

[33] A. Pazy, Semigroups of linear operators and applications to partial differential equations. Appl. Math. Sci. Springer-Verlag, New York 44 (1983).

[34] T. von Petersdorff and C. Schwab, Fully discrete multiscale Galerkin BEM, in Multiresolution Analysis and Partial Differential Equations, W. Dahmen, P. Kurdila and P. Oswald Eds., Academic Press, New York, Wavelet Anal. Appl. 6 (1997) $287-346$.

[35] K. Prause, The Generalized Hyperbolic Model: Estimation, Financial Derivatives, and Risk Measures. Ph.D. thesis AlbertLudwigs-Universität Freiburg i.Br. (1999). 
[36] P. Protter, Stochastic Integration and Differential Equations. Springer-Verlag (1990).

[37] S. Raible, Lévy processes in Finance: Theory, Numerics, and Empirical Facts. Ph.D. thesis Albert-Ludwigs-Universität Freiburg i.Br. (2000).

[38] K.-I. Sato, Lévy Processes and Infinitely Divisible Distributions. Cambridge University Press (1999).

[39] D. Schötzau and C. Schwab, hp-discontinuous Galerkin time-stepping for parabolic problems. C.R. Acad. Sci. Paris 333 (2001) 1121-1126.

[40] W. Schoutens, Lévy Processes in Finance. Wiley Ser. Probab. Stat., Wiley Publ. (2003).

[41] T. von Petersdorff and C. Schwab, Wavelet-discretizations of parabolic integro-differential equations. SIAM J. Numer. Anal. 41 (2003) 159-180.

[42] T. von Petersdorff and C. Schwab, Numerical solution of parabolic equations in high dimensions. Report NI03013-CPD, Isaac Newton Institute for the Mathematical Sciences, Cambridge, UK (2003), http://www.newton.cam.ac.uk/preprints2003.html, ESAIM: M2AN 38 (2004) 93-127.

[43] X. Zhang, Analyse Numerique des Options Américaines dans un Modèle de Diffusion avec Sauts. Ph.D. thesis, École Normale des Ponts et Chaussées (1994)

To access this journal online: www.edpsciences.org 